quatrième série-tome 41 fascicule 6 novembre-décembre 2008

$$
\begin{aligned}
& \text { ANNALES } \\
& \text { SCIENTIFIQUES } \\
& \text { de } \\
& \text { L'ÉCOLE } \\
& \text { NORMALE } \\
& \text { SUPÉRIEURE }
\end{aligned}
$$

Christophe MOUROUGANE \& Shigeharu TAKAYAMA

Hodge metrics and the curvature of higher direct images 


\title{
HODGE METRICS AND THE CURVATURE OF HIGHER DIRECT IMAGES
}

\author{
By Christophe MOUROUGANE and Shigeharu TAKAYAMA
}

\begin{abstract}
Using the harmonic theory developed by Takegoshi for representation of relative cohomology and the framework of computation of curvature of direct image bundles by Berndtsson, we prove that the higher direct images by a smooth morphism of the relative canonical bundle twisted by a semi-positive vector bundle are locally free and semi-positively curved, when endowed with a suitable Hodge type metric.
\end{abstract}

RÉSUMÉ. - Nous utilisons la théorie de représentation par formes harmoniques des classes de cohomologie relative développée par Takegoshi et la structure des calculs de courbure de fibrés images directes développée par Berndtsson, pour étudier les images directes supérieures par un morphisme lisse du fibré canonique relatif tensorisé par un fibré vectoriel holomorphe hermitien semi-positif. Nous montrons qu'elles sont localement libres et que, munies de métriques convenables de type Hodge, elles sont à courbure semi-positive.

\section{Introduction}

This is a continuation of our works [23] [24] on the metric positivity of direct image sheaves of adjoint bundles. The goal of this paper is to prove the following

Theorem 1.1. - Let $f: X \longrightarrow Y$ be a holomorphic map of complex manifolds, which is smooth, proper, Kähler, surjective, and with connected fibers. Let $(E, h)$ be a holomorphic vector bundle on $X$ with a Hermitian metric $h$ of semi-positive curvature in the sense of Nakano. Then for any $q \geq 0$, the direct image sheaf $R^{q} f_{*} \Omega_{X / Y}^{n}(E)$ is locally free and Nakano semipositive, where $n$ is the dimension of fibers.

A real $(1,1)$-form $\omega$ on $X$ is said to be a relative Kähler form for $f: X \longrightarrow Y$, if for every point $y \in Y$, there exists a local coordinate $\left(W ;\left(t_{1}, \ldots, t_{m}\right)\right)$ around $y$ such that $\omega+c f^{*}\left(\sqrt{-1} \sum_{j} d t_{j} \wedge d \overline{t_{j}}\right)$ is a Kähler form on $f^{-1}(W)$ for a constant $c$. A morphism $f$ is said to be Kähler, if there exists a relative Kähler form $\omega_{f}$ for $f$ (see [27, 6.1]). 
In case when $E$ is a semi-positive line bundle and $q=0$, Theorem 1.1 is a theorem of Berndtsson [2, 1.2]. In our previous paper [24], we obtained independently from [2] a weaker semi-positivity: the Griffiths semi-positivity of $f_{*} \Omega_{X / Y}^{n}(E)$ for a semi-ample line bundle $E$. Right after two papers [2] [24], especially [2] have appeared, the analogous statement for higher direct images has been considered as a next problem among others. Theorem 1.1 solves this problem for Nakano semi-positive vector bundles $E$.

For a Nakano semi-positive vector bundle $E$, the local freeness $R^{q} f_{*} \Omega_{X / Y}^{n}(E)$ is a consequence of Takegoshi's injectivity theorem [27]. Here is one point where we use the smoothness of $f$. We can only expect the torsion freeness in general, by Kollár [15], [16] ([27] in analytic setting). Another theorem in [27] shows that $R^{q} f_{*} \Omega_{X / Y}^{n}(E)$ can be embedded into $f_{*} \Omega_{X / Y}^{n-q}(E)$ at least locally on $Y$, and that $R^{q} f_{*} \Omega_{X / Y}^{n}(E)=0$ for $q>n$. The sheaf $f_{*} \Omega_{X / Y}^{n-q}(E)$ has a natural Hermitian metric induced from a relative Kähler form $\omega_{f}$ and $h$ (at least where it is locally free) the so-called Hodge metric. For local sections $\sigma, \tau \in H^{0}\left(W, f_{*} \Omega_{X / Y}^{n-q}(E)\right)$, the inner product at $y \in W \subset Y$ is given by $\int_{X_{y}}\left(\left.\sigma\right|_{X_{y}}\right) \wedge \bar{*}_{h_{y}}\left(\left.\tau\right|_{X_{y}}\right)$, where $X_{y}=f^{-1}(y)$ is the fiber, and $\bar{*}_{h_{y}}$ is the "star"-operator with respect to $\omega_{y}=\left.\omega_{f}\right|_{X_{y}}$ and $h_{y}=\left.h\right|_{X_{y}}$. By pulling back this Hodge metric via the injection, say $S_{f}^{q}: R^{q} f_{*} \Omega_{X / Y}^{n}(E) \longrightarrow f_{*} \Omega_{X / Y}^{n-q}(E)$, we have a Hermitian metric on $R^{q} f_{*} \Omega_{X / Y}^{n}(E)$ in the usual sense. Our original contribution is the right definition of this "Hodge metric" on $R^{q} f_{*} \Omega_{X / Y}^{n}(E)$, and our main theorem is that its curvature is Nakano semi-positive.

The space $H^{q}\left(X_{y}, \Omega_{X_{y}}^{n}\left(E_{y}\right)\right)$ has another natural inner product with respect to $\omega_{y}$ and $h_{y}$. For cohomology classes $u_{y}, v_{y} \in H^{q}\left(X_{y}, \Omega_{X_{y}}^{n}\left(E_{y}\right)\right)$, it is given by $\int_{X_{y}} u_{y}^{\prime} \wedge \bar{*}_{h_{y}} v_{y}^{\prime}$, where $u_{y}^{\prime}$ and $v_{y}^{\prime}$ are the harmonic representatives of $u_{y}$ and $v_{y}$ respectively. These fiberwise inner products also define a Hermitian metric on $R^{q} f_{*} \Omega_{X / Y}^{n}(E)$. We first tried to compute its curvature, but we did not succeed it.

We follow [2], not [24], for the method of computation of the curvature. Since one can directly see the original method in [2], let us explain the differences from [2], i.e., the differences in cases $q=0$ and $q>0$. In case $q=0$, the map $S_{f}^{0}: f_{*} \Omega_{X / Y}^{n}(E) \longrightarrow f_{*} \Omega_{X / Y}^{n}(E)$ is an isomorphism, in fact the multiplication by a constant. Moreover $f_{*} \Omega_{X / Y}^{n}(E)$ is locally free thanks to Ohsawa-Takegoshi type $L^{2}$-extension theorem [26][25][20], and the (1,0)derivative of $\sigma \in H^{0}\left(Y, f_{*} \Omega_{X / Y}^{n}(E)\right)=H^{0}\left(X, \Omega_{X / Y}^{n}(E)\right)$ vanishes on each fiber $X_{y}$ by simply a bidegree reason. However in case $q>0$, we have no local freeness of $f_{*} \Omega_{X / Y}^{n-q}(E)$, nor the vanishing of the $(1,0)$-derivative of $\sigma \in H^{0}\left(Y, f_{*} \Omega_{X / Y}^{n-q}(E)\right)=H^{0}\left(X, \Omega_{X / Y}^{n-q}(E)\right)$ on $X_{y}$. To overcome these difficulties, we need to restrict ourselves to consider the image of $S_{f}^{q}: R^{q} f_{*} \Omega_{X / Y}^{n}(E) \longrightarrow f_{*} \Omega_{X / Y}^{n-q}(E)$. Then we have a local freeness as we mentioned above, and the vanishing of the $(1,0)$-derivative thanks to an estimate by Takegoshi [27]. This is a key fact, and which is like the $d$-closedness of holomorphic $p$-forms on a compact Kähler manifold. After getting those key observations: the local freeness, the right Hodge metric to be considered, and the closedness of holomorphic sections, the computation of the curvature is a straightforward generalization of [2].

There are many positivity results of direct image sheaves of relative canonical bundles and of adjoint bundles, which are mostly about the positivity in algebraic geometry. We will recall only a few here. The origin is due to Griffiths in his theory on the variation of Hodge structures [9]. Griffiths' work has been generalized by Fujita [7], Kawamata [12], Viehweg 
[29], Kollár [15], [16] and so on, in more algebro-geometric setting. There are also positivity results on higher direct images by Moriwaki [22], Fujino [6] and so on. We refer to [21][5][30] [17] for further remarks on related results. On the analytic side, it can be understood as the plurisubharmonic variation of related functions to the Robin constant [32] [18], or of the Bergman kernels [19]. There is also a series of works by Yamaguchi. As he mentioned in [2], his method is inspired by those works. There are also related recent works by Berndtsson-Păun [3] and Tsuji [28].

Acknowledgments. We would like to thank Professor Berndtsson for his correspondences in many occasions, for answering questions, and showing us a revised version of his paper [2]. A part of this work was done during the second named author's stay in Rennes. He would like to thank the mathematical department of Rennes for a support to stay there.

\section{Preliminaries}

\subsection{Hermitian vector bundles}

Let $X$ be a complex manifold of dimension $n$ with a Hermitian metric $\omega$, and let $E$ be a holomorphic vector bundle of rank $r$ on $X$ with a Hermitian metric $h$. Let $\left(E^{*}, h^{*}\right)$ be the dual vector bundle. Let $A^{p, q}(X, E)$ be the space of $E$-valued smooth $(p, q)$-forms, and $A_{0}^{p, q}(X, E)$ be the space of $E$-valued smooth $(p, q)$-forms with compact support. Let $*: A^{p, q}(X, E) \longrightarrow A^{n-q, n-p}(X, E)$ be the Hodge star-operator with respect to $\omega$. For any $u \in A^{p, q}(X, E)$ and $v \in A^{s, t}(X, E)$, we define $u \wedge h \bar{v} \in A^{p+s, q+t}(X, \mathbb{C})$ as follows. We take a local trivialization of $E$ on an open subset $U \subset X$, and we regard $u={ }^{t}\left(u_{1}, \ldots, u_{r}\right)$ as a row vector with $(p, q)$-forms $u_{j}$ on $U$, and similarly for $v={ }^{t}\left(v_{1}, \ldots, v_{r}\right)$. The Hermitian metric $h$ is then a matrix valued function $h=\left(h_{j k}\right)$ on $U$. We define $u \wedge h \bar{v}$ locally on $U$ by

$$
u \wedge h \bar{v}=\sum_{j, k} u_{j} \wedge h_{j k} \overline{v_{k}} \in A^{p+s, q+t}(U, \mathbb{C}) .
$$

We should write ${ }^{t} u \wedge h \bar{v}$, but if there is no risk of confusions, we will write in this way. In this manner, we can define anti-linear isomorphisms $\sharp_{h}: A^{p, q}(X, E) \longrightarrow A^{q, p}\left(X, E^{*}\right)$ by $\sharp_{h} u=h \bar{u}$, and $\bar{*}_{h}=\sharp_{h} \circ *: A^{p, q}(X, E) \longrightarrow A^{n-p, n-q}\left(X, E^{*}\right)$ by $\bar{*}_{h} u=h \overline{* u}$. The inner product on $A_{0}^{p, q}(X, E)$ is defined by $(u, v)_{h}=\int_{X} u \wedge \bar{*}_{h} v$. Denote by $D_{h}=\partial_{h}+\bar{\partial}$ the metric connection, and by $\Theta_{h}=D_{h}^{2}$ the curvature of $(E, h)$. The Hermitian vector bundle $(E, h)$ is said to be Nakano semi-positive (resp. Nakano positive), if the End (E)-valued real $(1,1)$-from $\sqrt{-1} \Theta_{h}$ is positive semi-definite (resp. positive definite) quadratic form on each fiber of the vector bundle $T_{X} \otimes E$.

We define $\vartheta_{h}: A^{p, q}(X, E) \longrightarrow A^{p, q-1}(X, E)$ by $\vartheta_{h}=-* \partial_{h^{*}}=-\bar{*}_{h^{*}} \bar{\partial}_{\bar{*}_{h}}$, which is the formal adjoint operator of $\bar{\partial}: A^{p, q}(X, E) \longrightarrow A^{p, q+1}(X, E)$ with respect to the inner product $(,)_{h}$. We also define $\bar{\vartheta}: A^{p, q}(X, E) \longrightarrow A^{p-1, q}(X, E)$ by $\bar{\vartheta}=-* \bar{\partial} *$, which is the formal adjoint operator of $\partial_{h}: A^{p, q}(X, E) \longrightarrow A^{p+1, q}(X, E)$ with respect to the inner product $(,)_{h}$. We denote by $e(\theta)$ the left exterior product acting on $A^{p, q}(X, E)$ by a form $\theta \in A^{s, t}(X, \mathbb{C})$. Then the adjoint operator $e(\theta)^{*}$ with respect to the inner product $(,)_{h}$ is defined by $e(\theta)^{*}=(-1)^{(p+q)(s+t+1)} * e(\bar{\theta}) *$. For instance we set $\Lambda_{\omega}=e(\omega)^{*}$. We recall the following very useful relation $([11,1.2 .31][31,6.29])$ : 
Lemma 2.1. - For a primitive element $u \in A^{p, q}(X, E)$, i.e., $p+q=k \leq n$ and $\Lambda_{\omega} u=0$, the Hodge *-operator reads

$$
*\left(\omega^{j} \wedge u\right)=\sqrt{-1}^{p-q}(-1)^{\frac{k(k+1)}{2}} \frac{j !}{(n-k-j) !} \omega^{n-k-j} \wedge u
$$

for every $0 \leq j \leq n-k$.

As immediate consequences, we have

COROLlary 2.2. - Denote by $c_{n-q}=\sqrt{-1}^{(n-q)^{2}}=\sqrt{-1}^{n-q}(-1)^{(n-q)(n-q-1) / 2}$.

(1) Let $a, b \in A^{n-q, 0}(X, E)$. Then $* a=\left(\overline{c_{n-q}} / q !\right) \omega^{q} \wedge a,{ }^{*} h a=\left(c_{n-q} / q !\right) \omega^{q} \wedge h \bar{a}$, $a=\left(c_{n-q} / q !\right) *\left(\omega^{q} \wedge a\right)$, and $a \wedge \bar{*}_{h} b=\left(c_{n-q} / q !\right) \omega^{q} \wedge a \wedge h \bar{b}$.

(2) Let $u \in A^{n, q}(X, E)$. Then $u=\left(c_{n-q} / q !\right) \omega^{q} \wedge * u$.

Notation 2.3. - We use the following conventions often. Denote by $c_{d}=\sqrt{-1} d^{2}=$ $\sqrt{-1}^{d}(-1)^{d(d-1) / 2}$ for any non-negative integer $d$. Let $t=\left(t_{1}, \ldots, t_{m}\right)$ be the coordinates of $\mathbb{C}^{m}$.

(1) $d t=d t_{1} \wedge \cdots \wedge d t_{m}, d \bar{t}=\overline{d t}$, and $d V_{t}:=c_{m} d t \wedge d \bar{t}=\bigwedge_{j=1}^{m} \sqrt{-1} d t_{j} \wedge d \overline{t_{j}}>0$.

(2) Let $\widehat{d t_{j}}$ be a smooth $(m-1,0)$-form without $d t_{j}$ such that $d t_{j} \wedge \widehat{d t_{j}}=d t$, and $\widehat{d \bar{t}_{j}}=\widehat{\widehat{d t_{j}}}$.

(3) Let $\widehat{d t_{j} \wedge d t_{k}}$ be a smooth $(m-1, m-1)$-form without $d t_{j}$ and $d \overline{t_{k}}$ such that $\sqrt{-1} d t_{j} \wedge$ $d \overline{t_{k}} \wedge \overline{d t_{j} \wedge d \overline{t_{k}}}=c_{m} d t \wedge d \bar{t}$.

\subsection{Set up}

In the rest of this paper, we will use the following set up.

Let $X$ and $Y$ be complex manifolds of $\operatorname{dim} X=n+m$ and $\operatorname{dim} Y=m$. Let $f: X \longrightarrow Y$ be a holomorphic map, which is smooth, proper, Kähler, surjective, and with connected fibers. Let $(E, h)$ be a holomorphic vector bundle on $X$ of rank $r$, with a Hermitian metric $h$ whose curvature $\Theta_{h}$ is semi-positive in the sense of Nakano.

(I) a general setting: $f:\left(X, \omega_{f}\right) \longrightarrow Y$. We take a relative Kähler form $\omega_{f}$ for $f$, and let $\kappa_{f}=\left\{\omega_{f}\right\}$ be the de Rham cohomology class. On each fiber $X_{y}$, we have a Kähler form $\omega_{y}=\left.\omega_{f}\right|_{X_{y}}$, and a Nakano semi-positive vector bundle $\left(E_{y}, h_{y}\right)=\left.(E, h)\right|_{X_{y}}$.

(II) a localized setting of (I): $f:(X, \omega) \longrightarrow Y \subset \mathbb{C}^{m}$. We further assume that the base $Y$ is a unit ball in $\mathbb{C}^{m}$ with coordinates $t=\left(t_{1}, \ldots, t_{m}\right)$ and with admissible charts over $Y$ (see below). We take a global Kähler form $\omega=\omega_{f}+c f^{*}\left(\sqrt{-1} \sum d t_{j} \wedge d \overline{t_{j}}\right)$ on $X$ for large $c>0$, without changing the class $\kappa_{f}$ nor the fiberwise Kähler forms $\omega_{y}$.

Since $f: X \longrightarrow Y$ is smooth, for every point $y \in Y$, we can take a local coordinate $\left(W ; t=\left(t_{1}, \ldots, t_{m}\right)\right)$ centered at $y$, so that $(W ; t)$ is a unit ball in $\mathbb{C}^{m}$, and a system of local coordinates $\mathcal{U}=\left\{\left(U_{\alpha} ; z_{\alpha}, t\right) ; \alpha=1,2,3, \ldots\right\}$ of $f^{-1}(W)$ which is locally finite, and every $U_{\alpha}$ is biholomorphic to a product $D_{\alpha} \times W$ for a domain $D_{\alpha}$ in $\mathbb{C}^{n}$; $x \mapsto\left(z_{\alpha}^{1}(x), \ldots, z_{\alpha}^{n}(x), t_{1}, \ldots, t_{m}\right)$, namely the projection from $D_{\alpha} \times W$ to $W$ is compatible with the map $\left.f\right|_{U_{\alpha}}$. We can write $z_{\alpha}^{j}=f_{\alpha \beta}^{j}\left(z_{\beta}^{1}, \ldots, z_{\beta}^{n}, t_{1}, \ldots, t_{m}\right)$ for $1 \leq j \leq n$ on $U_{\alpha} \cap U_{\beta}$. All $f_{\alpha \beta}^{j}\left(z_{\beta}, t\right)$ are holomorphic in $z_{\beta}$ and $t$. We call it admissible charts $\mathcal{U}$ over $W$ (cf. [14, $\S 2.3])$.

Since our assertions are basically local on $Y$, we will mostly use the set up (II). The set up (I) will be used in subsections 3.1, 3.3, 4.3 and 5.1. 


\section{Generalities of relative differential forms}

Let $f:\left(X, \omega_{f}\right) \longrightarrow Y$ and $(E, h)$ be as in $\S 2.2$.I. We recall the complex analytic properties of the relative cotangent bundle $\Omega_{X / Y}=\Omega_{X} / f^{*} \Omega_{Y}$ and the bundle of relative holomorphic $p$-forms $\Omega_{X / Y}^{p}=\bigwedge^{p} \Omega_{X / Y}$. We will not distinguish a vector bundle and the corresponding locally free sheaf. For a subset $S \subset Y$, we denote by $X_{S}=f^{-1}(S)$ and $E_{S}=\left.E\right|_{X_{S}}$.

\subsection{Definition of relative differential forms}

Let $U \subset X$ be an open subset.

(1) For a form $u \in A^{p, q}(U, E)$, we have the restriction $\left.u\right|_{X_{y} \cap U} \in A^{p, q}\left(X_{y} \cap U, E\right)$ on each fiber over $y \in Y$, which is the pull-back as a form via the inclusion $X_{y} \longrightarrow X$. Two forms $u, v \in A^{p, q}(U, E)$ are said to be $f$-equivalent " $u \sim v$ ", if $\left.u\right|_{X_{y} \cap U}=\left.v\right|_{X_{y} \cap U}$ for any $t \in Y$. We denote the set of equivalence classes by

$$
A^{p, q}(U / Y, E)=A^{p, q}(U, E) / \sim .
$$

The set $A^{p, q}(U / Y, E)$ will be called the space of relative differential forms on $U$. We denote by $[u] \in A^{p, q}(U / Y, E)$ the equivalence class of $u \in A^{p, q}(U, E)$.

(2) A form $u \in A^{p, 0}(U, E)$ is said to be holomorphic on each fiber, if the restriction $\left.u\right|_{X_{y}}$ is holomorphic, i.e., $\left.u\right|_{X_{y}} \in H^{0}\left(X_{y}, \Omega_{X_{y}}^{p}\left(E_{y}\right)\right)$ for every $y \in Y$. A form $u \in A^{p, 0}(U, E)$ is said to be relatively holomorphic, if for any local chart $\left(W ; t=\left(t_{1}, \ldots, t_{m}\right)\right)$ of $Y$, the form $u \wedge f^{*} d t$ is holomorphic on $X_{W} \cap U$, i.e., $u \wedge f^{*} d t \in H^{0}\left(X_{W} \cap U, \Omega_{X}^{p+m}(E)\right)$.

(3) For a function $\alpha \in A^{0}(Y, \mathbb{C})$ and $[u] \in A^{p, 0}(U / Y, E)$, we can define $\alpha[u]:=$ $\left[\left(f^{*} \alpha\right) u\right] \in A^{p, 0}(U / Y, E)$. For each open subset $W \subset Y$, we set

$$
\begin{aligned}
& A^{0}\left(W, f_{*} \Omega_{X / Y}^{p}(E)\right):=\left\{[u] \in A^{p, 0}\left(X_{W} / W, E\right) ; u \text { is holomorphic on each fiber }\right\}, \\
& H^{0}\left(W, f_{*} \Omega_{X / Y}^{p}(E)\right):=\left\{[u] \in A^{p, 0}\left(X_{W} / W, E\right) ; u \text { is relatively holomorphic }\right\} .
\end{aligned}
$$

We can see that $A^{0}\left(W, f_{*} \Omega_{X / Y}^{p}(E)\right)$ becomes an $A^{0}(W, \mathbb{C})$-module, and $H^{0}\left(W, f_{*} \Omega_{X / Y}^{p}(E)\right)$ becomes an $H^{0}\left(W, \mathcal{O}_{W}\right)$-module.

(4) It is sometimes convenient to use local coordinates to look at those properties above. Let $u \in A^{p, q}(X, E)$. On an admissible chart $\left(U_{\alpha} ; z_{\alpha}, t\right)$ as above, we can write

$$
u=\sum_{I \in I_{p}, J \in I_{q}} u_{I J \alpha} d z_{\alpha}^{I} \wedge \overline{d z_{\alpha}^{J}}+R
$$

where $u_{I J \alpha}=u_{I J \alpha}\left(z_{\alpha}, t\right) \in A^{0}\left(U_{\alpha}, \mathbb{C}^{r}\right)$, and

$$
R \in \sum_{j} A^{p-1, q}\left(U_{\alpha}, \mathbb{C}^{r}\right) \wedge d t_{j}+\sum_{j} A^{p, q-1}\left(U_{\alpha}, \mathbb{C}^{r}\right) \wedge d \overline{t_{j}}
$$

Here we use a standard convention. We set $I_{p}=\left\{\left\{i_{1}, i_{2}, \ldots, i_{p}\right\} ; 1 \leq i_{1}<i_{2}<\cdots<i_{p} \leq n\right\}$, and $I_{0}$ is empty. For $I=\left\{i_{1}, i_{2}, \ldots, i_{p}\right\} \in I_{p}$, we set $d z_{\alpha}^{I}=d z_{\alpha}^{i_{1}} \wedge \cdots \wedge d z_{\alpha}^{i_{p}}$. Similar for $J \in I_{q}$ and $d \overline{z_{\alpha}^{J}}$. The restriction on a fiber is locally given by

$$
\left.u\right|_{X_{y}}=\left.\sum_{I \in I_{p}, J \in I_{q}} u_{I J \alpha}\right|_{X_{y}} d z_{\alpha}^{I} \wedge \overline{d z_{\alpha}^{J}}
$$

In particular, for two forms $u, v \in A^{p, q}(X, E)$, they are $f$-equivalent $u \sim v$ if and only if $u_{I J \alpha}=v_{I J \alpha}$ for any $(I, J) \in I_{p} \times I_{q}$ on any admissible chart $\left(U_{\alpha} ; z_{\alpha}, t\right)$. 
(5) Let $u \in A^{p, 0}(X, E)$. On an admissible chart $\left(U_{\alpha} ; z_{\alpha}, t\right)$, we have

$$
u=\sum_{I \in I_{p}} u_{I \alpha} d z_{\alpha}^{I}+R
$$

as above. Therefore $u$ is holomorphic on each fiber (resp. relatively holomorphic) if and only if every $u_{I \alpha}$ is holomorphic in $z_{\alpha}$ (resp. holomorphic in $z_{\alpha}$ and $t$ ) for any $I \in I_{p}$ on any admissible chart $\left(U_{\alpha} ; z_{\alpha}, t\right)$.

\subsection{Holomorphic structure of $f_{*} \Omega_{X / Y}^{p}(E)$}

We also give the holomorphic structure on $f_{*} \Omega_{X / Y}^{p}(E)$ by an action of the $\bar{\partial}$-operator. Let $\left(W ;\left(t_{1}, \ldots, t_{m}\right)\right) \subset Y$ be a local chart, over which we have admissible charts. Let $[\sigma] \in A^{0}\left(W, f_{*} \Omega_{X / Y}^{p}(E)\right)$, which can be seen as a differentiable family of holomorphic forms. Since $\left.(\bar{\partial} \sigma)\right|_{X_{y}}=\bar{\partial}\left(\left.\sigma\right|_{X_{y}}\right)=0$, we can write

$$
\bar{\partial} \sigma=\sum_{j} \eta^{j} \wedge d t_{j}+\sum_{j} \nu^{j} \wedge d \overline{t_{j}}
$$

with some $\eta^{j} \in A^{p-1,1}\left(X_{W}, E\right)$ and some $\nu^{j} \in A^{p, 0}\left(X_{W}, E\right)$. In particular $\bar{\partial}(\sigma \wedge$ $d t)=\sum_{j} \nu^{j} \wedge d \overline{t_{j}} \wedge d t$. On an admissible chart $(U ; z, t)=\left(U_{\alpha} ; z_{\alpha}, t\right)$, we write $\sigma=\sum_{I \in I_{p}} \sigma_{I} d z^{I}+R$ with $R \in \sum_{j} A^{p-1,0}\left(U_{\alpha}, \mathbb{C}^{r}\right) \wedge d t_{j}$. Then we have $\left.\nu^{j}\right|_{X_{y} \cap U}=$ $\left.(-1)^{p} \sum_{I \in I_{p}}\left(\partial \sigma_{I} / \partial \overline{t_{j}}\right)\right|_{X_{y} \cap U} d z^{I} \in H^{0}\left(X_{y} \cap U, \Omega_{X_{y}}^{p}\left(E_{y}\right)\right)$ for every $j$. In particular, the class $\left[\nu^{j}\right] \in A^{0}\left(W, f_{*} \Omega_{X / Y}^{p}(E)\right)$ is well-defined for $[\sigma]$. For $[\sigma] \in A^{0}\left(W, f_{*} \Omega_{X / Y}^{p}(E)\right)$, we define

$$
\bar{\partial}[\sigma]=\sum_{j}\left[\nu^{j}\right] d \overline{t_{j}} \in A^{0,1}\left(W, f_{*} \Omega_{X / Y}^{p}(E)\right) .
$$

Here $A^{0,1}\left(W, f_{*} \Omega_{X / Y}^{p}(E)\right)=A^{0}\left(W, f_{*} \Omega_{X / Y}^{p}(E)\right) \otimes A^{0,1}(W, \mathbb{C})$ as $A^{0}(W, \mathbb{C})$-module, but it has only a formal meaning. Then, $[\sigma] \in H^{0}\left(W, f_{*} \Omega_{X / Y}^{p}(E)\right)$ if and only if $\bar{\partial}[\sigma] \equiv 0$. In fact both of them are characterized by the holomorphicity of all $\sigma_{I}$ in $z$ and $t$ locally.

Lemma 3.1. - Let $\left(W ;\left(t_{1}, \ldots, t_{m}\right)\right) \subset Y$ be a local chart as above in $\$ 2.2$. Let $\sigma \in$ $A^{p, 0}\left(X_{W}, E\right)$ such that $[\sigma] \in H^{0}\left(W, f_{*} \Omega_{X / Y}^{p}(E)\right)$. Then (1)

$$
\bar{\partial} \sigma=\sum_{j} \eta^{j} \wedge d t_{j}
$$

with some $\eta^{j} \in A^{p-1,1}\left(X_{W}, E\right)$,

(2) these $\eta^{j}$ are not unique, but $\left[\eta^{j}\right] \in A^{p-1,1}\left(X_{W} / W, E\right)$ are well-defined for $\sigma$,

(3) all $\left.\eta^{j}\right|_{X_{y}}$ are $\bar{\partial}$-closed on any $X_{y}$, and

(4) [2, Lemma 4.3] all $\left.\eta^{j}\right|_{X_{y}} \wedge \omega_{y}^{q+1}$ are $\bar{\partial}$-exact on any $X_{y}$.

Proof. - (1) is now clear. We show (2) and (3). For each $j, \sigma \wedge \widehat{d t_{j}} \in A^{p+m-1,0}\left(X_{W}, E\right)$ is well-defined for $\sigma$, and so is $\bar{\partial}\left(\sigma \wedge \widehat{d t_{j}}\right)=\eta^{j} \wedge d t$. Hence $\left[\eta^{j}\right]$ are well-defined for $\sigma$, by Remark 3.2 below. Moreover $\left(\bar{\partial} \eta^{j}\right) \wedge d t=\bar{\partial} \bar{\partial}\left(\sigma \wedge \widehat{d t_{j}}\right)=0$. Hence we obtain $\bar{\partial}\left(\left.\eta^{j}\right|_{X_{y}}\right)=$ $\left.\left(\bar{\partial} \eta^{j}\right)\right|_{X_{y}}=0$ by Remark 3.2 again.

(4) We fix $j$. By a bidegree reason, we can write $\sigma \wedge \widehat{d t_{j}} \wedge \omega^{q+1}=a^{j} \wedge d t$ with some $a^{j} \in$ $A^{n, q+1}(X, E)$. We note that the class $\left[a^{j}\right] \in A^{n, q+1}(X / Y, E)$ is well-defined by Remark 3.2. 
By taking $\bar{\partial}$, we have $\eta^{j} \wedge d t \wedge \omega^{q+1}=\left(\bar{\partial} a^{j}\right) \wedge d t$. Then $\left[\eta^{j} \wedge \omega^{q+1}\right]=\left[\bar{\partial} a^{j}\right]$ in $A^{n, q+2}(X / Y, E)$ by Remark 3.2, and hence $\left.\eta^{j}\right|_{X_{y}} \wedge \omega_{y}^{q+1}=\bar{\partial}\left(\left.a^{j}\right|_{X_{y}}\right)$ on any $X_{y}$.

Remark 3.2. - For $u, v \in A^{p, q}\left(X_{W}, E\right)$, a relation $u \wedge d t=v \wedge d t$ implies $[u]=[v]$ in $A^{p, q}\left(X_{W} / W, E\right)$, and the converse holds true in case $q=0$.

REMARK 3.3. - Each cohomology class $\left\{\left.\eta^{j}\right|_{X_{y}}\right\} \in H^{p-1,1}\left(X_{y}, E_{y}\right)$ is well-defined for $[\sigma] \in H^{0}\left(W, f_{*} \Omega_{X / Y}^{p}(E)\right)$. As it is well-known, $\left\{\left.\eta^{j}\right|_{X_{y}}\right\}$ is obtained by the cup-product (up to a sign) with the Kodaira-Spencer class of $f: X \longrightarrow Y$ at $y \in Y$ for $t_{j}$-direction. However we will not use these remarks.

\subsection{Canonical pairing}

There is a canonical pairing on each stalk $f_{*} \Omega_{X / Y}^{p}(E)_{y}$ with respect to $\omega_{y}$ and $h_{y}$, via the natural inclusion $f_{*} \Omega_{X / Y}^{p}(E)_{y} \subset H^{0}\left(X_{y}, \Omega_{X_{y}}^{p}\left(E_{y}\right)\right)$. At each point $y \in Y$, we have the fiberwise inner product

$$
g_{y}\left(\sigma_{y}, \tau_{y}\right):=\left(\sigma_{y}, \tau_{y}\right)_{h_{y}}=\int_{X_{y}}\left(c_{p} /(n-p) !\right) \omega_{y}^{n-p} \wedge \sigma_{y} \wedge h \overline{\tau_{y}}
$$

for $\sigma_{y}, \tau_{y} \in A^{p, 0}\left(X_{y}, E_{y}\right)$. When considered as germs $\sigma_{y}, \tau_{y} \in f_{*} \Omega_{X / Y}^{p}(E)_{y}$, we will denote this inner product by $g_{y}\left(\sigma_{y}, \tau_{y}\right)$. On the other hand, when considered as forms $\sigma_{y}, \tau_{y} \in$ $H^{0}\left(X_{y}, \Omega_{X_{y}}^{p}\left(E_{y}\right)\right)$, we will denote it by $\left(\sigma_{y}, \tau_{y}\right)_{h_{y}}$. These two are the same, but our standing points are different, i.e., at a point $y \in Y$, or on the fiber $X_{y}$.

For relative forms $[\sigma],[\tau] \in A^{p, 0}\left(X_{W} / W, E\right)$ (or $H^{0}\left(W, f_{*} \Omega_{X / Y}^{p}(E)\right)$ ) over an open subset $W \subset Y$, the above fiberwise inner product gives

$$
g([\sigma],[\tau]):=f_{*}\left(\left(c_{p} /(n-p) !\right) \omega_{f}^{n-p} \wedge \sigma \wedge h \bar{\tau}\right),
$$

where the right hand side is a push-forward as a current. For a test $(m, m)$-form $\varphi$ on $W$ (i.e., a smooth form with compact support), we have $f_{*}\left(\left(c_{p} /(n-p) !\right) \omega_{f}^{n-p} \wedge \sigma \wedge h \bar{\tau}\right)(\varphi):=$ $\int_{X}\left(c_{p} /(n-p) !\right) \omega_{f}^{n-p} \wedge \sigma \wedge h \bar{\tau} \wedge f^{*} \varphi$. Hence the right hand side does not depend on representatives $\sigma$ nor $\tau$ (see Remark 3.2). Since the map $f$ is smooth, $g([\sigma],[\tau])$ is in fact a smooth function on $W$.

We remark that the definition of the pairing $g$ depends only on the fiberwise Kähler forms $\left\{\omega_{y}\right\}_{y \in Y}$. For example, over a local chart $(W ; t) \subset Y$, we can replace $\omega_{f}$ by $\omega_{f}+c f^{*}\left(\sqrt{-1} \sum d t_{j} \wedge d \overline{t_{j}}\right)$ for any $c \in \mathbb{R}$ in the definition of $g([\sigma],[\tau])$. The pairing $g$ defines a Hermitian metric on every locally free subsheaf of $f_{*} \Omega_{X / Y}^{p}(E)$ in the usual sense, which we call the Hodge metric. As a matter of fact, $g$ itself is called the Hodge metric on $f_{*} \Omega_{X / Y}^{p}(E)$ commonly, although it may not be locally free.

\section{Harmonic theory for Nakano semi-positive vector bundles}

We collect some fundamental results of Takegoshi [27], and immediate consequences from them. 


\subsection{Absolute setting}

Let $\left(X_{0}, \omega_{0}\right)$ be an $n$-dimensional compact Kähler manifold and let $\left(E_{0}, h_{0}\right)$ be a holomorphic Hermitian vector bundle on $X_{0}$. We have an inner product $(,)_{h_{0}}$ and the associated norm \|\|$_{h_{0}}$ on each $A^{p, q}\left(X_{0}, E_{0}\right)$. Let $\mathcal{H}^{p, q}\left(X_{0}, E_{0}\right)$ be the space of harmonic $(p, q)$-forms. From Bochner-Kodaira-Nakano formula, it then follows that if $\left(E_{0}, h_{0}\right)$ is furthermore Nakano semi-positive and Nakano positive at one point, then $H^{q}\left(X_{0}, \Omega_{X_{0}}^{n}\left(E_{0}\right)\right)$ vanish for all $q>0$.

Enoki [4] and Takegoshi [27] (a special case of Theorem 4.1 below) show that if $\left(E_{0}, h_{0}\right)$ is Nakano semi-positive, then the Hodge $*$-operator yields injective homomorphism $*_{0}: \mathcal{H}^{n, q}\left(X_{0}, E_{0}\right) \longrightarrow H^{0}\left(X_{0}, \Omega_{X_{0}}^{n-q}\left(E_{0}\right)\right)$. Recalling that $\left(c_{n-q} / q !\right) \omega_{0}^{q} \wedge *_{0} u=u$ for $u \in A^{n, q}\left(X_{0}, E_{0}\right)$, it then follows that the Lefschetz operator $L_{0}^{q}: H^{0}\left(X_{0}, \Omega_{X_{0}}^{n-q}\left(E_{0}\right)\right) \longrightarrow$ $H^{q}\left(X_{0}, \Omega_{X_{0}}^{n}\left(E_{0}\right)\right)$ is surjective. Hence we have

$$
H^{0}\left(X_{0}, \Omega_{X_{0}}^{n-q}\left(E_{0}\right)\right)=\operatorname{Ker} L_{0}^{q} \oplus *_{0} \mathcal{H}^{n, q}\left(X_{0}, E_{0}\right) .
$$

\subsection{Localized relative setting}

Let $f:(X, \omega) \longrightarrow Y \subset \mathbb{C}^{m}$ and $(E, h)$ be as in $\S 2.2$.II. We take a $C^{\infty}$ plurisubharmonic exhaustion function $\Phi=f^{*} \sum_{j=1}^{m}\left|t_{j}\right|^{2}$ on $X$. We take any $0 \leq q \leq n$. Following [27, 4.3], we set the following subspace of $E$-valued harmonic $(n+m, q)$-forms with respect to $\omega$ and $h$ :

$$
\mathcal{H}^{n+m, q}(X, E, \Phi)=\left\{u \in A^{n+m, q}(X, E) ; \bar{\partial} u=\vartheta_{h} u=0 \text { and } e(\bar{\partial} \Phi)^{*} u=0 \text { on } X\right\} .
$$

By [27, 4.3.i], $u \in \mathcal{H}^{n+m, q}(X, E, \Phi)$ if and only if $\bar{\vartheta} u=0,\left(\sqrt{-1} e\left(\Theta_{h}+\partial \bar{\partial} \Phi\right) \Lambda_{\omega} u\right) \wedge h \bar{u}=0$ and $e(\bar{\partial} \Phi)^{*} u=0$ on $X$. One can check easily that $\left(f^{*} \alpha\right) u$ satisfies those latter three conditions, if $\alpha \in H^{0}\left(Y, \mathcal{O}_{Y}\right)$ and if $u \in \mathcal{H}^{n+m, q}(X, E, \Phi)$.

Theorem $4.1([27,4.3])$. - (1) The space $\mathcal{H}^{n+m, q}(X, E, \Phi)$ does not depend on $C^{\infty}$ plurisubharmonic exhaustion functions $\Phi$, and has a natural structure of $H^{0}\left(Y, \mathcal{O}_{Y}\right)$-module.

(2) For $u \in \mathcal{H}^{n+m, q}(X, E, \Phi)$, one has $\bar{\partial} * u=0$ and $\partial_{h} * u=0$. In particular, the Hodge *-operator yields an injective homomorphism $*: \mathcal{H}^{n+m, q}(X, E, \Phi) \longrightarrow H^{0}\left(X, \Omega_{X}^{n+m-q}(E)\right)$, and $\mathcal{H}^{n+m, q}(X, E, \Phi)$ becomes a torsion free $H^{0}\left(Y, \mathcal{O}_{Y}\right)$-module.

Let $\iota^{\prime}: Z_{\bar{\partial}}^{n+m, q}(X, E) \longrightarrow H^{q}\left(X, \Omega_{X}^{n+m}(E)\right)$ be the quotient map which induces the Dolbeault's isomorphism.

Theorem $4.2([27,5.2])$. - (1) The space $\mathcal{H}^{n+m, q}(X, E, \Phi)$ represents $H^{q}\left(X, \Omega_{X}^{n+m}(E)\right)$ as a torsion free $H^{0}\left(Y, \mathcal{O}_{Y}\right)$-module, in particular the quotient map $\iota^{\prime}$ induces an isomorphism $\iota: \mathcal{H}^{n+m, q}(X, E, \Phi) \longrightarrow H^{q}\left(X, \Omega_{X}^{n+m}(E)\right)$.

(2) The injective homomorphism * : $\mathcal{H}^{n+m, q}(X, E, \Phi) \longrightarrow H^{0}\left(X, \Omega_{X}^{n+m-q}(E)\right)$ induces a splitting homomorphism (up to a constant)

$$
* \circ \iota^{-1}: H^{q}\left(X, \Omega_{X}^{n+m}(E)\right) \longrightarrow H^{0}\left(X, \Omega_{X}^{n+m-q}(E)\right)
$$

for the Lefschetz homomorphism

$$
L^{q}: H^{0}\left(X, \Omega_{X}^{n+m-q}(E)\right) \longrightarrow H^{q}\left(X, \Omega_{X}^{n+m}(E)\right) .
$$

such that $\left(c_{n+m-q} / q !\right) L^{q} \circ * \circ \iota^{-1}=i d$.

(3) Let $u \in \mathcal{H}^{n+m, q}(X, E, \Phi)$. Then the form $* u \in H^{0}\left(X, \Omega_{X}^{n+m-q}(E)\right)$ is saturated in base variables, i.e., $* u=\sigma_{u} \wedge d t$ for some $\left[\sigma_{u}\right] \in H^{0}\left(X, \Omega_{X / Y}^{n-q}(E)\right.$ ) (see the proof of $[27$, 
5.2.ii]). In particular, $u=\left(c_{n+m-q} / q !\right) \omega^{q} \wedge \sigma_{u} \wedge d t$ and the map $u \mapsto\left[\sigma_{u}\right]$ is well-defined. Thus the Hodge *-operator induces an injective homomorphism

$$
S^{q}: \mathcal{H}^{n+m, q}(X, E, \Phi) \longrightarrow H^{0}\left(X, \Omega_{X / Y}^{n-q}(E)\right) .
$$

In Theorem 4.2(3), we used our assumption that $f$ is smooth.

We take a trivialization $\mathcal{O}_{Y} \simeq \Omega_{Y}^{m}$ given by $1 \mapsto d t$, which induces isomorphisms of sheaves $\Omega_{X / Y}^{n} \cong \Omega_{X / Y}^{n} \otimes f^{*} \Omega_{Y}^{m} \cong \Omega_{X}^{n+m}$ by $[u] \mapsto u \wedge d t$, and hence of cohomology groups $\alpha^{q}: H^{q}\left(X, \Omega_{X / Y}^{n}(E)\right) \simeq H^{q}\left(X, \Omega_{X}^{n+m}(E)\right)$. We also have an injection $\Omega_{X / Y}^{n-q} \longrightarrow \Omega_{X}^{n+m-q}$ by $[\sigma] \mapsto \sigma \wedge d t$, and hence an injection $\beta^{0}: H^{0}\left(X, \Omega_{X / Y}^{n-q}(E)\right) \longrightarrow H^{0}\left(X, \Omega_{X}^{n+m-q}(E)\right)$. Combining with Theorem 4.2, we have

$$
\begin{aligned}
& \iota^{-1} \circ \alpha^{q}: H^{q}\left(X, \Omega_{X / Y}^{n}(E)\right) \longrightarrow H^{q}\left(X, \Omega_{X}^{n+m}(E)\right) \simeq \mathcal{H}^{n+m, q}(X, E, \Phi), \\
& *=\beta^{0} \circ S^{q}: \mathcal{H}^{n+m, q}(X, E, \Phi) \longrightarrow H^{0}\left(X, \Omega_{X / Y}^{n-q}(E)\right) \longrightarrow H^{0}\left(X, \Omega_{X}^{n+m-q}(E)\right), \\
&\left(\alpha^{q}\right)^{-1} \circ L^{q}: H^{0}\left(X, \Omega_{X}^{n+m-q}(E)\right) \longrightarrow H^{q}\left(X, \Omega_{X}^{n+m}(E)\right) \simeq H^{q}\left(X, \Omega_{X / Y}^{n}(E)\right) .
\end{aligned}
$$

Then Theorem 4.2 (2) reads the following relative version:

Corollary 4.3. - Let

$$
\begin{aligned}
& S_{f}^{q}=S^{q} \circ \iota^{-1} \circ \alpha^{q}: H^{q}\left(X, \Omega_{X / Y}^{n}(E)\right) \longrightarrow H^{0}\left(X, \Omega_{X / Y}^{n-q}(E)\right), \\
& L_{f}^{q}=\left(\alpha^{q}\right)^{-1} \circ L^{q} \circ \beta^{0}: H^{0}\left(X, \Omega_{X / Y}^{n-q}(E)\right) \longrightarrow H^{q}\left(X, \Omega_{X / Y}^{n}(E)\right) .
\end{aligned}
$$

Then $\left(c_{n+m-q} / q !\right) L_{f}^{q} \circ S_{f}^{q}=i d$ on $H^{q}\left(X, \Omega_{X / Y}^{n}(E)\right)$.

We can also see, thanks to [27, 5.2.iv] (see also [27, 6.5.i]) that those constructions can be localized on $Y$, and induce homomorphisms of direct image sheaves.

COROLlaRY 4.4. - There exist homomorphisms induced from the Hodge *-operator and the Lefschetz homomorphism:

$$
S_{f}^{q}: R^{q} f_{*} \Omega_{X / Y}^{n}(E) \longrightarrow f_{*} \Omega_{X / Y}^{n-q}(E), \quad L_{f}^{q}: f_{*} \Omega_{X / Y}^{n-q}(E) \longrightarrow R^{q} f_{*} \Omega_{X / Y}^{n}(E)
$$

so that $\left(c_{n+m-q} / q\right.$ !) $L_{f}^{q} \circ S_{f}^{q}=i d$ on $R^{q} f_{*} \Omega_{X / Y}^{n}(E)$. In particular

$$
f_{*} \Omega_{X / Y}^{n-q}(E)=F^{n-q} \oplus \mathcal{K}^{n-q} \text {, with } F^{n-q}=\operatorname{Im} S_{f}^{q} \text { and } \mathcal{K}^{n-q}=\operatorname{Ker} L_{f}^{q} .
$$

We translate some results above into explicite forms.

Lemma 4.5. - Let $\sigma \in A^{n-q, 0}(X, E)$ such that $[\sigma] \in H^{0}\left(Y, F^{n-q}\right)$. Then

$$
\partial_{h} \sigma=\sum_{j} \mu^{j} \wedge d t_{j}
$$

for some $\mu^{j} \in A^{n-q, 0}(X, E)$,

(2) these $\mu^{j}$ are not unique, but $\left[\mu^{j}\right] \in A^{n-q, 0}(X / Y, E)$ are well-defined for $\sigma$, and

(3) $\partial_{h_{y}}\left(\left.\mu^{j}\right|_{X_{y}}\right)=0$ on any $X_{y}$ and all $j$.

Proof. - There exists $u \in \mathcal{H}^{n+m, q}(X, E, \Phi)$ such that $* u=\sigma \wedge d t \in H^{0}\left(X, \Omega_{X}^{n+m-q}(E)\right)$. By Takegoshi's Theorem 4.1, we have $\partial_{h} * u=0$. Hence $\left(\partial_{h} \sigma\right) \wedge d t=\partial_{h} * u=0$, and we have (1). We can show (2) and (3) by the same method in Lemma 3.1. 
REMARK 4.6. - Unlike in the case $q=0$ that is treated by degree considerations [2, §4], we used the semi-positivity here. In general, for $[\sigma] \in H^{0}\left(Y, f_{*} \Omega_{X / Y}^{n-q}(E)\right)$ with $q>0$, we cannot have $\partial_{h} \sigma=\sum \mu^{j} \wedge d t_{j}$ for some $\mu^{j} \in A^{n-q, 0}(X, E)$. This is in fact a key property, and it makes various computations possible. We also note that $\eta^{j}$ in Lemma 3.1 and $\mu^{j}$ are not well-defined for a class $[\sigma] \in H^{0}\left(Y, F^{n-q}\right)$, and that means, we have some freedom of choices in a class.

Stalks or fibers at a point $y \in Y$ will be denoted by $f_{*} \Omega_{X / Y}^{n-q}(E)_{y}, F_{y}^{n-q}, \mathcal{K}_{y}^{n-q}$ respectively. Those stalks can be seen as subspaces of $H^{0}\left(X_{y}, \Omega_{X_{y}}^{n-q}\left(E_{y}\right)\right)$, i.e., $F_{y}^{n-q} \oplus \mathcal{K}_{y}^{n-q}=$ $f_{*} \Omega_{X / Y}^{n-q}(E)_{y} \subset H^{0}\left(X_{y}, \Omega_{X_{y}}^{n-q}\left(E_{y}\right)\right)$.

Lemma 4.7. - Let $\sigma_{y} \in F_{y}^{n-q}$ and $\tau_{y} \in \mathcal{K}_{y}^{n-q}$, and regard them as elements of $H^{0}\left(X_{y}, \Omega_{X_{y}}^{n-q}\left(E_{y}\right)\right)$. Then, (1) $\partial_{h_{y}} \sigma_{y}=0$ in $A^{n-q+1,0}\left(X_{y}, E_{y}\right)$,

(2) $\omega_{y}^{q} \wedge \tau_{y} \in A^{n, q}\left(X_{y}, E_{y}\right)$ is $\bar{\partial}$-exact, and

(3) $\left(\sigma_{y}, \tau_{y}\right)_{h_{y}}=\int_{X_{y}}\left(c_{n-q} / q !\right) \omega_{y}^{q} \wedge \sigma_{y} \wedge h_{y} \overline{\tau_{y}}=0$.

Proof. - We will argue at $y=0$.

(1) Since $Y$ is a unit ball in $\mathbb{C}^{m}$, there exists $[\sigma] \in H^{0}\left(Y, F^{n-q}\right)$ such that $\left.\sigma\right|_{X_{0}}=\sigma_{0}$. By Lemma 4.5, we have $\partial_{h_{0}}\left(\left.\sigma\right|_{X_{0}}\right)=0$.

(2) We take $[\tau] \in H^{0}\left(Y, \mathcal{K}^{n-q}\right)$ such that $\left.\tau\right|_{X_{0}}=\tau_{0}$. We have $L_{f}^{q}([\tau])=0$. Recall the definition of $L_{f}^{q}=\left(\alpha^{q}\right)^{-1} \circ L^{q} \circ \beta^{0}$, where $\beta^{0}([\tau])=\tau \wedge d t$, and $\left(\alpha^{q}\right)^{-1}$ is an isomorphism. Then we have $L^{q} \circ \beta^{0}([\tau])=0$ in $H^{q}\left(X, \Omega_{X}^{n+m}(E)\right)$, namely $\omega^{q} \wedge \tau \wedge d t=\bar{\partial} a$ for some $a \in A^{n+m, q-1}(X, E)$. By a bidegree reason, $a$ can be written as $a=b \wedge d t$ for some $b \in$ $A^{n, q-1}(X, E)$. Then $\left(\omega^{q} \wedge \tau-\bar{\partial} b\right) \wedge d t=0$. By restricting on $X_{0}$, we have $\omega_{0}^{q} \wedge \tau_{0}-\bar{\partial} b_{0}=0$, where $b_{0}=\left.b\right|_{X_{0}}$.

(3) By (2), we have $\int_{X_{0}} \omega_{0}^{q} \wedge \sigma_{0} \wedge h_{0} \overline{\tau_{0}}=\int_{X_{0}} \sigma_{0} \wedge h_{0} \overline{\bar{\partial} b_{0}}$. Since $\partial\left(\sigma_{0} \wedge h_{0} \overline{b_{0}}\right)=$ $\left(\partial_{h_{0}} \sigma_{0}\right) \wedge h_{0} \overline{b_{0}}+(-1)^{n-q} \sigma_{0} \wedge h_{0} \overline{\bar{\partial} b_{0}}$, and since $\partial_{h_{0}} \sigma_{0}=0$ by (1), we have $\int_{X_{0}} \sigma_{0} \wedge h_{0} \overline{\bar{\partial} b_{0}}=$ $(-1)^{n-q} \int_{X_{0}} \partial\left(\sigma_{0} \wedge h_{0} \overline{\bar{\partial} b_{0}}\right)=0$.

\subsection{Local freeness}

We shall show that the direct image sheaves $R^{q} f_{*} \Omega_{X / Y}^{n}(E)$ are locally free. This is an immediate consequence of a result of Takegoshi [27]. We start by recalling a general remark.

Lemma 4.8. - Let $X$ and $Y$ be varieties (reduced and irreducible), $f: X \longrightarrow Y$ be a proper surjective morphism, and let $\mathcal{E}$ be a coherent sheaf on $X$ which is flat over $Y$. Assume that the natural map $\varphi^{q}(y): R^{q} f_{*} \mathcal{E} \otimes \mathbb{C}(y) \longrightarrow H^{q}\left(X_{y}, \mathcal{E}_{y}\right)$ is surjective for any $y \in Y$ and any $q \geq 0$, where $X_{y}$ is the fiber over $y$, and $\mathcal{E}_{y}$ is the induced sheaf ([10, III.9.4]). Then $R^{q} f_{*} \mathcal{E}$ is locally free for any $q \geq 0$, and $\varphi^{q}(y): R^{q} f_{*} \mathcal{E} \otimes \mathbb{C}(y) \longrightarrow H^{q}\left(X_{y}, \mathcal{E}_{y}\right)$ is an isomorphism for any $y \in Y$ and any $q \geq 0$.

Proof. - By [10, III.12.11(a)] (cohomology and base change), the surjectivity of $\varphi^{q}(y)$ implies that it is an isomorphism. By [10, III.12.11(b)], the local freeness of $R^{q} f_{*} \mathcal{E}$ in a neighborhood of $y \in Y$ follows from the surjectivities of $\varphi^{q}(y)$ and of $\varphi^{q-1}(y)$.

We can find the corresponding results in the category of complex spaces, for example [1, III.3.4, III.3.7]. 
Lemma 4.9 (cf. [27, 6.8]). - Let $f:\left(X, \omega_{f}\right) \longrightarrow Y$ and $(E, h)$ be as in \$2.2.I. Then

(1) the natural restriction map $R^{q} f_{*} \Omega_{X / Y}^{n}(E) \longrightarrow H^{q}\left(X_{y}, \Omega_{X_{y}}^{n}\left(E_{y}\right)\right)$ is surjective for any $y \in Y$ and any $q \geq 0$, and

(2) $R^{q} f_{*} \Omega_{X / Y}^{n}(E)$ is locally free for any $q \geq 0$, and $\varphi^{q}(y): R^{q} f_{*} \Omega_{X / Y}^{n}(E) \otimes \mathbb{C}(y) \longrightarrow$ $H^{q}\left(X_{y}, \Omega_{X_{y}}^{n}\left(E_{y}\right)\right)$ is an isomorphism for any $y \in Y$ and any $q \geq 0$.

Proof. - (1) Fix $y \in Y$. Since our assertion is local on $Y$, we may assume that $Y$ is a unit ball in $\mathbb{C}^{m}$ with coordinates $t=\left(t_{1}, \ldots, t_{m}\right)$ centered at $y=\{t=0\}$. We take a trivialization $\mathcal{O}_{Y} \cong \Omega_{Y}^{m}$ given by $1 \mapsto d t=d t_{1} \wedge \cdots \wedge d t_{m}$. For every $i$ with $1 \leq i \leq m$, we let $Y_{i}=\left\{t_{1}=\cdots=t_{i}=0\right\}$ be a complex sub-manifold of $Y, X_{i}=f^{-1}\left(Y_{i}\right)$, and let $f_{i}: X_{i} \longrightarrow Y_{i}$ be the induced morphism. We set $X_{0}=X, Y_{0}=Y$ and $f_{0}=f$. By the injectivity theorem of Takegoshi with $F=\mathcal{O}_{X}$ in [27, 6.8.i], the sheaf homomorphism $R^{q} f_{0 *}\left(f_{0}^{*} t_{1}\right): R^{q} f_{*} \Omega_{X / Y}^{n}(E) \otimes \Omega_{Y}^{m} \longrightarrow R^{q} f_{*} \Omega_{X / Y}^{n}(E) \otimes \Omega_{Y}^{m}$ induced by the product with the holomorphic function $f^{*} t_{1}$ is injective for any $q \geq 0$. Hence the restriction map $R^{q} f_{0 *} \Omega_{X / Y}^{n}(E) \longrightarrow R^{q} f_{1 *}\left(\Omega_{X / Y}^{n}(E) \otimes \mathcal{O}_{X_{1}}\right)$ is surjective for any $q \geq 0$. By the adjunction formula, we have $\Omega_{X / Y}^{n} \otimes \mathcal{O}_{X_{1}}=\Omega_{X_{1} / Y_{1}}^{n}$. Hence inductively, we obtain a surjection $R^{q} f_{*} \Omega_{X / Y}^{n}(E) \longrightarrow H^{q}\left(X_{y}, \Omega_{X_{y}}^{n}\left(E_{y}\right)\right)$.

(2) This follows from (1) and Lemma 4.8.

\section{The Hodge metric}

We shall define a canonical Hermitian metric on $R^{q} f_{*} \Omega_{X / Y}^{n}(E)$, and compute the metric connection and the curvature. $\$ 5.1$ will be discussed in the global setting $\$ 2.2$.I, and the rest of this section will be discussed in the localized setting §2.2.II.

\subsection{Definition of Hodge metrics}

We define a canonical Hermitian metric on a vector bundle $R^{q} f_{*} \Omega_{X / Y}^{n}(E)$, which we call the Hodge metric.

Definition 5.1. - Let $f:\left(X, \omega_{f}\right) \longrightarrow Y$ and $(E, h)$ be as in $\S 2.2 . I$, and let $0 \leq q \leq n$. For every point $y \in Y$, we take a local coordinate $W \cong\left\{t \in \mathbb{C}^{m} ;\|t\|<1\right\}$ centered at $y$, and a Kähler form $\omega=\omega_{f}+c f^{*}\left(\sqrt{-1} \sum d t_{j} \wedge d \overline{t_{j}}\right)$ on $X_{W}$ for a real number $c$. A choice of a Kähler form $\omega$ gives an injection $S_{\omega}:=S_{f}^{q}: R^{q} f_{*} \Omega_{X / Y}^{n}(E) \longrightarrow f_{*} \Omega_{X / Y}^{n-q}(E)$ over $W$ (Corollary 4.4). Then for every pair of vectors $u_{y}, v_{y} \in R^{q} f_{*} \Omega_{X / Y}^{n}(E)_{y}$, we define

$$
g\left(u_{y}, v_{y}\right)=\left.\int_{X_{y}}\left(c_{n-q} / q !\right)\left(\omega_{f}^{q} \wedge S_{\omega}\left(u_{y}\right) \wedge h \overline{S_{\omega}\left(v_{y}\right)}\right)\right|_{X_{y}} .
$$

Here the right hand side is the restriction on the image of $S_{\omega}$ of the canonical pairing, say $g$ again, on $f_{*} \Omega_{X / Y}^{n-q}(E)_{y}$ in $\S 3.3$.

The injection $S_{\omega}=S_{f}^{q}: R^{q} f_{*} \Omega_{X / Y}^{n}(E) \longrightarrow f_{*} \Omega_{X / Y}^{n-q}(E)$ over $W$ may depend on the choices of Kähler forms in the relative Kähler class $\left\{\omega_{f}\right\}$, however 
Lemma 5.2. - In the situation in Definition 5.1, the induced metric $g$ on $\left.R^{q} f_{*} \Omega_{X / Y}^{n}(E)\right|_{W}$ via $S_{\omega}=S_{f}^{q}: R^{q} f_{*} \Omega_{X / Y}^{n}(E) \longrightarrow f_{*} \Omega_{X / Y}^{n-q}(E)$ over $W$ does not depend on the choice of a Kähler form $\omega$ in the relative Kähler class $\left\{\left.\omega_{f}\right|_{X_{W}}\right\} \in H^{2}\left(X_{W}, \mathbb{R}\right)$ so that $\left.\omega\right|_{X_{y}}=\left.\omega_{f}\right|_{X_{y}}$ for any $y \in W$, and hence $g$ defines a global Hermitian metric on $R^{q} f_{*} \Omega_{X / Y}^{n}(E)$ over $Y$ by varying $y \in Y$.

Proof. - It is enough to check it in case $u_{y}=v_{y}$. We may also assume that $Y=W \subset \mathbb{C}^{m}$. We take two Kähler forms $\omega_{1}$ and $\omega_{2}$ on $X$, which relate $\omega_{1}-\omega_{2}=f^{*} \sqrt{-1} \partial \bar{\partial} \psi$ for some $\psi \in A^{0}(Y, \mathbb{R})$.

(i) We need to recall the definition of $S_{f}^{q}$. Let

$$
u \in H^{0}\left(Y, R^{q} f_{*} \Omega_{X / Y}^{n}(E)\right) \cong H^{q}\left(X, \Omega_{X / Y}^{n}(E)\right)
$$

be an extension of $u_{y}$. With respect to $\omega_{i}$, we denote by $*_{i}$ the Hodge $*$-operator, by $\mathcal{H}^{n+m, q}\left(X, \omega_{i}, E, \Phi\right)$ the space of harmonic forms in Theorem 4.1 with $\Phi=f^{*}\|t\|^{2}$, by $\iota_{i}: \mathcal{H}^{n+m, q}\left(X, \omega_{i}, E, \Phi\right) \simeq H^{q}\left(X, \Omega_{X}^{n+m}(E)\right)$ the isomorphism in Theorem 4.2(1), and by $S_{i}$ the injection $S_{f}^{q}: R^{q} f_{*} \Omega_{X / Y}^{n}(E) \longrightarrow f_{*} \Omega_{X / Y}^{n-q}(E)$. We have isomorphisms

$$
\mathcal{H}_{i}=\iota_{i}^{-1} \circ \alpha^{q}: H^{q}\left(X, \Omega_{X / Y}^{n}(E)\right) \simeq \mathcal{H}^{n+m, q}\left(X, \omega_{i}, E, \Phi\right)
$$

(see the argument before Corollary 4.3). Then we have $*_{i} \mathcal{H}_{i}(u) \in H^{0}\left(X, \Omega_{X}^{n+m-q}(E)\right.$ ), and $*_{i} \mathcal{H}_{i}(u)=\sigma_{i} \wedge d t$ for some $\left[\sigma_{i}\right] \in H^{0}\left(X, \Omega_{X / Y}^{n-q}(E)\right)$. Namely $S_{i}(u)=\left[\sigma_{i}\right]$. In this setting, our lemma is reduced to show that

$$
f_{*}\left(\omega_{1}^{q} \wedge \sigma_{1} \wedge h \overline{\sigma_{1}}\right)=f_{*}\left(\omega_{2}^{q} \wedge \sigma_{2} \wedge h \overline{\sigma_{2}}\right) .
$$

This is reduced to show $\int_{X}\left(f^{*} \beta\right) \omega_{1}^{q} \wedge \sigma_{1} \wedge d t \wedge h \overline{\sigma_{1} \wedge d t}=\int_{X}\left(f^{*} \beta\right) \omega_{2}^{q} \wedge \sigma_{2} \wedge d t \wedge h \overline{\sigma_{2} \wedge d t}$ for any $\beta \in A^{0}(Y, \mathbb{C})$ with compact support. We take such a $\beta \in A^{0}(Y, \mathbb{C})$.

(ii) Since the Dolbeault cohomology classes of $\mathcal{H}_{1}(u)$ and $\mathcal{H}_{2}(u)$ are the same, there exists $a \in A^{n+m, q-1}(X, E)$ such that $\mathcal{H}_{1}(u)-\mathcal{H}_{2}(u)=\left(c_{n+m-q} / q !\right) \bar{\partial} a$. Recalling Corollary $2.2(2)$ that $\mathcal{H}_{i}(u)=\left(c_{n+m-q} / q !\right) \omega_{i}^{q} \wedge *_{i} \mathcal{H}_{i}(u)$, we have $\omega_{1}^{q} \wedge *_{1} \mathcal{H}_{1}(u)-\omega_{2}^{q} \wedge *_{2} \mathcal{H}_{2}(u)=\bar{\partial} a$, and hence $\omega_{1}^{q} \wedge \sigma_{1} \wedge d t-\omega_{2}^{q} \wedge \sigma_{2} \wedge d t=\bar{\partial} a$.

By a degree reason in the base variables, we have $f^{*}(\bar{\partial} \beta) \wedge d \bar{t}=0$. Hence $\bar{\partial}\left(\left(f^{*} \beta\right) a \wedge\right.$ $\left.h \overline{\sigma_{1} \wedge d t}\right)=\left(f^{*} \beta\right) \bar{\partial} a \wedge h \overline{\sigma_{1} \wedge d t}+(-1)^{n+m+q-1}\left(f^{*} \beta\right) a \wedge h \overline{\partial_{h}\left(\sigma_{1} \wedge d t\right)}$. We also have $\partial_{h}\left(\sigma_{1} \wedge\right.$ $d t)=\partial_{h} *_{1} \mathcal{H}_{1}(u)=0$ by Theorem 4.1 (2). Hence $\int_{X}\left(f^{*} \beta\right) \bar{\partial} a \wedge h \overline{\sigma_{1} \wedge d t}=\int_{X} \bar{\partial}\left(\left(f^{*} \beta\right) a \wedge\right.$ $\left.h \overline{\sigma_{1} \wedge d t}\right)=0$ by the Stokes theorem. Then the relation $\omega_{1}^{q} \wedge \sigma_{1} \wedge d t=\omega_{2}^{q} \wedge \sigma_{2} \wedge d t+\bar{\partial} a$ implies that $\int_{X}\left(f^{*} \beta\right) \omega_{1}^{q} \wedge \sigma_{1} \wedge d t \wedge h \overline{\sigma_{1} \wedge d t}=\int_{X}\left(f^{*} \beta\right) \omega_{2}^{q} \wedge \sigma_{2} \wedge d t \wedge h \overline{\sigma_{1} \wedge d t}$.

(iii) Now we use $\omega_{1}-\omega_{2}=f^{*} \sqrt{-1} \partial \bar{\partial} \psi$. This leads $\int_{X}\left(f^{*} \beta\right) \omega_{2}^{q} \wedge \sigma_{2} \wedge d t \wedge h \overline{\sigma_{1} \wedge d t}=$ $\int_{X}\left(f^{*} \beta\right) \omega_{1}^{q} \wedge \sigma_{2} \wedge d t \wedge h \overline{\sigma_{1} \wedge d t}$. The last integral equals to $\int_{X}\left(f^{*} \beta\right) \sigma_{2} \wedge d t \wedge h \overline{\omega_{1}^{q} \wedge \sigma_{1} \wedge d t}=$ $\int_{X}\left(f^{*} \beta\right) \sigma_{2} \wedge d t \wedge h \overline{\omega_{2}^{q} \wedge \sigma_{2} \wedge d t}+\int_{X}\left(f^{*} \beta\right) \sigma_{2} \wedge d t \wedge h \overline{\bar{\partial} a}$. By a similar manner as above, mainly because of $\partial_{h}\left(\sigma_{2} \wedge d t\right)=\partial_{h} *_{2} \mathcal{H}_{2}(u)=0$, we can see $\int_{X}\left(f^{*} \beta\right) \sigma_{2} \wedge d t \wedge h \overline{\bar{\partial} a}=0$. We finally obtain $\int_{X}\left(f^{*} \beta\right) \omega_{1}^{q} \wedge \sigma_{1} \wedge d t \wedge h \overline{\sigma_{1} \wedge d t}=\int_{X}\left(f^{*} \beta\right) \omega_{2}^{q} \wedge \sigma_{2} \wedge d t \wedge h \overline{\sigma_{2} \wedge d t}$.

At this point, we have the so-called metric connection (or the Chern connection) $D_{g}$ of the Hermitian vector bundle $\left(R^{q} f_{*} \Omega_{X / Y}^{n}(E), g\right)$, and the curvature $\Theta_{g}=D_{g}^{2}$. Since the curvature property in Theorem 1.1 is a local question on the base $Y$, it is enough to consider the following setting: 
Let $f:(X, \omega) \longrightarrow Y \subset \mathbb{C}^{m}$ and $(E, h)$ be as in $\S 2.2$.II, and let $0 \leq q \leq n$. We denote by $F=F^{n-q}$ the image of $S_{f}^{q}: R^{q} f_{*} \Omega_{X / Y}^{n}(E) \longrightarrow f_{*} \Omega_{X / Y}^{n-q}(E)$ with respect to $\omega$. Since, by definition, the canonical pairing $g$ on $f_{*} \Omega_{X / Y}^{n-q}(E)$ gives our $\left(R^{q} f_{*} \Omega_{X / Y}^{n}(E), g\right)$, we say a subbundle

$$
(F, g) \subset\left(f_{*} \Omega_{X / Y}^{n-q}(E), g\right)
$$

\subsection{The metric connection}

We shall construct the metric connection $D_{g}$ of $(F, g)$. Recall, Lemma 4.5, that $\partial_{h} \sigma=\sum_{j} \mu^{j} \wedge d t_{j}$ with some $\mu^{j} \in A^{n-q, 0}(X, E)$ for $[\sigma] \in H^{0}(Y, F)$. Since $A^{0}(Y, F)=$ $A^{0}(Y, \mathbb{C}) \otimes H^{0}(Y, F)$ as $A^{0}(Y, \mathbb{C})$-module, this formula holds for $[\sigma] \in A^{0}(Y, F)$, too. We consider the fiberwise orthogonal projection $P_{y}: A^{n-q, 0}\left(X_{y}, E_{y}\right) \longrightarrow F_{y}$ given by $u_{y} \mapsto \sum_{j=1}^{\ell} g_{y}\left(u_{y}, \sigma_{j y}\right) \sigma_{j y}$, where $\sigma_{1 y}, \ldots, \sigma_{\ell y} \in F_{y}$ is a basis of $F_{y}$. Since $F$ is locally free, the family $\left\{P_{y}\right\}_{y \in Y}$ induces a map

$$
P: A^{n-q, 0}(X, E) \longrightarrow\left\{u \in A^{n-q, 0}(X, E) ;\left.u\right|_{X_{y}} \in F_{y} \text { for any } y \in Y\right\} .
$$

Then for $[\sigma] \in A^{0}(Y, F)$ with $\partial_{h} \sigma=\sum_{j} \mu^{j} \wedge d t_{j}$, we define

$$
\partial_{g}[\sigma]=\sum\left[P\left(\mu^{j}\right)\right] d t_{j} \in A^{1,0}(Y, F) .
$$

Lemma 5.3. - The class $\left[P\left(\mu^{j}\right)\right]$ is well-defined for $[\sigma] \in A^{0}(Y, F)$.

Proof. - (1) We shall show that $\left.\mu^{j}\right|_{X_{y}}$ are perpendicular to $H^{0}\left(X_{y}, \Omega_{X_{y}}^{n-q}\left(E_{y}\right)\right)$ under the condition $[\sigma]=[0]$, namely $\left.\sigma\right|_{X_{y}}=0$ for any $y \in Y$. We write $\sigma=\sum \sigma_{j} \wedge d t_{j}$ with some $\sigma_{j} \in$ $A^{n-q-1,0}(X, E)$. We note that we can take $\mu^{j}=\partial_{h} \sigma_{j}$. We take any $s \in H^{0}\left(X_{y}, \Omega_{X_{y}}^{n-q}\left(E_{y}\right)\right)$. Then $\partial\left(\left.\omega_{y}^{q} \wedge \sigma_{j}\right|_{X_{y}} \wedge h_{y} \bar{s}\right)=\omega_{y}^{q} \wedge \partial_{h_{y}}\left(\left.\sigma_{j}\right|_{X_{y}}\right) \wedge h_{y} \bar{s}+\left.(-1)^{n-q} \omega_{y}^{q} \wedge \sigma_{j}\right|_{X_{y}} \wedge h_{y} \overline{\bar{\partial} s}$. Because of $\bar{\partial} s=0$, we have $g_{y}\left(\left.\left(\partial_{h} \sigma_{j}\right)\right|_{X_{y}}, s\right)=\left(c_{n-q} / q !\right) \int_{X_{y}} \omega_{y}^{q} \wedge \partial_{h_{y}}\left(\left.\sigma_{j}\right|_{X_{y}}\right) \wedge h_{y} \bar{s}=$ $\left(c_{n-q} / q !\right) \int_{X_{y}} \partial\left(\left.\omega_{y}^{q} \wedge \sigma_{j}\right|_{X_{y}} \wedge h_{y} \bar{s}\right)=0$.

(2) The above (1) is enough to show that $\left[P\left(\mu^{j}\right)\right]$ is well-defined. But in fact, (1) said slightly more.

Lemma 5.4. - The sum $D_{g}:=\partial_{g}+\bar{\partial}$ is the metric connection of the Hermitian vector bundle $(F, g)$.

Proof. - It is not difficult to see that it is a connection. Let us check the compatibility with the metric $g$. Let $[\sigma],[\tau] \in H^{0}(Y, F)$, and write $\partial_{h} \tau=\sum_{j} \mu^{j}(\tau) \wedge d t_{j}$. Then $\bar{\partial} g([\sigma],[\tau])=(-1)^{n-q} f_{*}\left(\left(c_{n-q} / q !\right) \omega^{q} \wedge \sigma \wedge h \overline{\partial_{h} \tau}\right)=\sum_{j} f_{*}\left(\left(c_{n-q} / q !\right) \omega^{q} \wedge \sigma \wedge h \overline{\mu^{j}(\tau)}\right) d \overline{t_{j}}$. Since $\left.\sigma\right|_{X_{y}} \in F_{y}$, the last term becomes $\sum_{j} f_{*}\left(\left(c_{n-q} / q !\right) \omega^{q} \wedge \sigma \wedge h \overline{P\left(\mu^{j}(\tau)\right)}\right) d \overline{t_{j}}$, and it is $\sum_{j} g\left([\sigma],\left[P\left(\mu^{j}(\tau)\right)\right]\right) d \overline{t_{j}}$. In the notation of $\S 2.1$, we can write as $g([\sigma],[\tau])=[\sigma] \wedge g \overline{[\tau]}$ and $\bar{\partial} g([\sigma],[\tau])=[\sigma] \wedge g \overline{\partial_{g}[\tau]}$. 


\subsection{Curvature formula}

We describe the Nakano semi-positivity of a Hermitian holomorphic vector bundle. Since it is a local property, we will discuss on a local chart. Let $Y \subset \mathbb{C}^{m}$ be a unit ball centered at 0 with coordinates $t=\left(t_{1}, \ldots, t_{m}\right)$, and let $F=Y \times \mathbb{C}^{\ell}$ be a trivial vector bundle with a non-trivial Hermitian metric $g$. (This $(F, g)$ may not necessarily be our original bundle.) We write $\Theta_{g}=\sum \Theta_{j k} d t_{j} \wedge d \overline{t_{k}}$ with $\Theta_{j k} \in \operatorname{End}(Y, F)$. Then $(F, g)$ is Nakano semi-positive at $t=0$, if and only if for any tensor $s=\sum_{j=1}^{m} \partial / \partial t_{j} \otimes \sigma_{j}^{0} \in\left(T_{Y} \otimes F\right)_{0}$, we have $\Theta_{g}(s)=$ $\sum_{j, k} g_{0}\left(\Theta_{j k} \sigma_{j}^{0}, \sigma_{k}^{0}\right) \geq 0$. Moreover the last quantity can be obtained another way from local sections. If $\sigma, \tau \in H^{0}(Y, F)$, we have $\frac{\partial^{2}}{\partial t_{j} \partial \overline{t_{k}}} g(\sigma, \tau)=g\left(\left(\partial_{g} \sigma\right)^{j},\left(\partial_{g} \tau\right)^{k}\right)-g\left(\Theta_{j k} \sigma, \tau\right)$, where $\partial_{g} \sigma=\sum_{j}\left(\partial_{g} \sigma\right)^{j} d t_{j} \in A^{1,0}(Y, F)$ and so on. Hence if $\sigma$ and $\tau$ are normal at 0 with respect to $g$ (i.e., $\partial_{g} \sigma=\partial_{g} \tau=0$ at 0 ), we have $\left.\left(\frac{\partial^{2}}{\partial t_{j} \partial \bar{t}_{k}} g(\sigma, \tau)\right)\right|_{t=0}=-g_{0}\left(\left.\Theta_{j k} \sigma\right|_{t=0},\left.\tau\right|_{t=0}\right)$.

Notation 5.5. - (1) Let $V$ be a continuous $(m, m)$-form on $Y \subset \mathbb{C}^{m}$. Then we can write $V=v(t) d V_{t}$ with a unique continuous function $v$ on $Y$, and we define $V_{t=0}:=v(0)$.

(2) Associated to $m$-ple $\sigma_{1}, \ldots, \sigma_{m} \in H^{0}(Y, F)$, we let

$$
T(\sigma)=\sum_{j, k} g\left(\sigma_{j}, \sigma_{k}\right) \widehat{d t_{j} \wedge d \bar{t}_{k}} \in A^{m-1, m-1}(Y, \mathbb{C}) .
$$

In case all $\sigma_{j}$ are normal at $t=0$, we have $\sqrt{-1} \partial \bar{\partial} T(\sigma)_{t=0}=-\sum_{j, k} g_{0}\left(\left.\Theta_{j k} \sigma_{j}\right|_{x_{0}},\left.\sigma_{k}\right|_{x_{0}}\right)$. Hence we have

Lemma $5.6([2, \S 2]) .-A$ Hermitian vector bundle $(F, g)$ on an open subset $Y \subset \mathbb{C}^{m}$ is Nakano semi-positive at $t=0$, if for any $m$-ple vectors $\sigma_{1}^{0}, \ldots, \sigma_{m}^{0} \in F_{0}$, there exist extensions $\sigma_{j} \in H^{0}(Y, F)$ of $\sigma_{j}^{0}$, all of which are normal at $t=0$ and satisfy $\sqrt{-1} \partial \bar{\partial} T(\sigma)_{t=0} \leq 0$.

We go back to our original situation. We prepare the following notations.

Notation 5.7. - Let $f:(X, \omega) \longrightarrow Y \subset \mathbb{C}^{m}$ and $(E, h)$ be as in $\$ 2.2$. II. Let $\sigma_{1}, \ldots, \sigma_{m} \in A^{n-q, 0}(X, E)$ such that $\left[\sigma_{j}\right] \in H^{0}(Y, F)$ for all $j$.

(1) We set

$$
\widehat{\sigma}=\sum \sigma_{j} \wedge \widehat{d t_{j}} \in A^{n-q+m-1,0}(X, E)
$$

Then

$$
T([\sigma])=\sum_{j, k} g\left(\left[\sigma_{j}\right],\left[\sigma_{k}\right]\right) \overline{d t_{j} \wedge d \overline{t_{k}}}=f_{*}\left(\left(c_{N} / q !\right) \omega^{q} \wedge \widehat{\sigma} \wedge h \overline{\widehat{\sigma}}\right) .
$$

Here $N=n-q+m-1$.

(2) We write $\partial_{h} \sigma_{j}=\sum_{k} \mu_{j}^{k} \wedge d t_{k}$. Then

$$
\partial_{h} \widehat{\sigma}=\sum_{j} \mu_{j}^{j} \wedge d t=: \mu \wedge d t
$$

with $\mu \in A^{n-q, 0}(X, E)$, or rather $[\mu] \in A^{n-q, 0}(X / Y, E)$.

(3) We write $\bar{\partial} \sigma_{j}=\sum_{k} \eta_{j}^{k} \wedge d t_{k}$. Then

$$
\bar{\partial} \widehat{\sigma}=\sum_{j} \eta_{j}^{j} \wedge d t=: \eta \wedge d t
$$

with $\eta \in A^{n-q-1,1}(X, E)$, or rather $[\eta] \in A^{n-q-1,1}(X / Y, E)$. 
Lemma 5.8 (cf. [2, (4.4)]). - In Notation 5.7, one has

$$
\begin{aligned}
-\sqrt{-1} \partial \bar{\partial} T([\sigma])_{t=0}= & f_{*}\left(\left(c_{N} / q !\right) \omega^{q} \wedge \sqrt{-1} \Theta_{h} \wedge \widehat{\sigma} \wedge h \overline{\widehat{\sigma}}\right)_{t=0} \\
& -\left.\int_{X_{0}}\left(c_{n-q} / q !\right)\left(\omega^{q} \wedge \mu \wedge h \bar{\mu}\right)\right|_{X_{0}}-\left.\int_{X_{0}}\left(c_{n-q} / q !\right)\left(\omega^{q} \wedge \eta \wedge h \bar{\eta}\right)\right|_{X_{0}} .
\end{aligned}
$$

REMARK 5.9. - The first term comes from the curvature of $E$, and contributes positively.

The second term is $-\left\|\left.\mu\right|_{X_{0}}\right\|_{h_{0}}^{2}$, and it can be seen as the "second fundamental form" of $F \subset \bigcup_{t \in Y} A^{n-q, 0}\left(X_{y}, E_{y}\right)$ at $t=0$. This negative contribution will be eliminated by a careful choice of forms $\sigma_{j}$, in $\S 6.2$.

The third term is not a definite form. In general one can write $\left.\eta\right|_{X_{0}}$ as a sum $\left.\eta\right|_{X_{0}}=\eta_{0}^{\prime}+$ $\omega_{0} \wedge \eta_{0}^{\prime \prime}$ for primitive forms $\eta_{0}^{\prime}$ and $\eta_{0}^{\prime \prime}$ on $X_{0}$, and then $-\left.\int_{X_{0}}\left(c_{n-q} / q !\right)\left(\omega^{q} \wedge \eta \wedge h \bar{\eta}\right)\right|_{X_{0}}=$ $\left\|\eta_{0}^{\prime}\right\|_{h_{0}}^{2}-\left\|\eta_{0}^{\prime \prime}\right\|_{h_{0}}^{2}$. In $\S 6.2$, we will show that we can take $\sigma_{j}$ so that all $\left.\eta_{j}^{k}\right|_{X_{0}}$ and hence $\left.\eta\right|_{X_{0}}$ are primitive on $X_{0}$. In that case, the third term is $-\left.\int_{X_{0}}\left(c_{n-q} / q !\right)\left(\omega^{q} \wedge \eta \wedge h \bar{\eta}\right)\right|_{X_{0}}=\left\|\left.\eta\right|_{X_{0}}\right\|_{h_{0}}^{2} \geq 0$. We should read the Kodaira-Spencer class contributes positively.

Proof of Lemma 5.8. - The proof will be done by direct computations. We first note that $f_{*}\left(\omega^{q} \wedge \bar{\partial} \widehat{\sigma} \wedge h \overline{\widehat{\sigma}}\right)=f_{*}\left(\omega^{q} \wedge \eta \wedge d t \wedge h \overline{\widehat{\sigma}}\right)=0$ as an $(m-1, m)$-current on $Y$, because it contains $d t$. By the same reason, we have $f_{*}\left(\omega^{q} \wedge \widehat{\sigma} \wedge h \overline{\bar{\partial}} \widehat{\sigma}\right)=0$, and hence, by taking $\bar{\partial}$, we have $f_{*}\left(\omega^{q} \wedge \widehat{\sigma} \wedge h \overline{\partial_{h} \bar{\partial} \widehat{\sigma}}\right)=-(-1)^{N} f_{*}\left(\omega^{q} \wedge \bar{\partial} \widehat{\sigma} \wedge h \overline{\bar{\partial} \widehat{\sigma}}\right)$. Then we have $\bar{\partial} f_{*}\left(\omega^{q} \wedge \widehat{\sigma} \wedge h \overline{\widehat{\sigma}}\right)=$ $(-1)^{N} f_{*}\left(\omega^{q} \wedge \widehat{\sigma} \wedge h \overline{\partial_{h} \widehat{\sigma}}\right)$, and then

$$
\partial \bar{\partial} f_{*}\left(\omega^{q} \wedge \widehat{\sigma} \wedge h \overline{\widehat{\sigma}}\right)=(-1)^{N} f_{*}\left(\omega^{q} \wedge \partial_{h} \widehat{\sigma} \wedge h \overline{\partial_{h} \widehat{\sigma}}\right)+f_{*}\left(\omega^{q} \wedge \widehat{\sigma} \wedge h \overline{\bar{\partial} \partial_{h} \widehat{\sigma}}\right) .
$$

Since $\partial_{h} \bar{\partial}+\bar{\partial} \partial_{h}=e\left(\Theta_{h}\right)$, we have $f_{*}\left(\omega^{q} \wedge \widehat{\sigma} \wedge h \overline{\bar{\partial}} \partial_{h} \widehat{\sigma}\right)=f_{*}\left(\omega^{q} \wedge \widehat{\sigma} \wedge h \overline{\Theta_{h} \wedge \widehat{\sigma}}\right)-f_{*}\left(\omega^{q} \wedge\right.$ $\left.\widehat{\sigma} \wedge h \overline{\partial_{h} \bar{\partial} \widehat{\sigma}}\right)$. Using $f_{*}\left(\omega^{q} \wedge \widehat{\sigma} \wedge h \overline{\partial_{h} \bar{\partial} \widehat{\sigma}}\right)=-(-1)^{N} f_{*}\left(\omega^{q} \wedge \bar{\partial} \widehat{\sigma} \wedge h \overline{\bar{\partial}} \widehat{\sigma}\right)$, we can write

$$
\begin{aligned}
\partial \bar{\partial} f_{*}\left(\omega^{q} \wedge \widehat{\sigma} \wedge h \bar{\sigma}\right)=-f_{*}\left(\omega^{q} \wedge \Theta_{h} \wedge \widehat{\sigma} \wedge h \overline{\widehat{\sigma}}\right) & +(-1)^{N+(n-q) m} f_{*}\left(\omega^{q} \wedge \mu \wedge h \bar{\mu} \wedge d t \wedge d \bar{t}\right) \\
& +(-1)^{N+(n-q) m} f_{*}\left(\omega^{q} \wedge \eta \wedge h \bar{\eta} \wedge d t \wedge d \bar{t}\right) .
\end{aligned}
$$

Here we mind that $\sqrt{-1} \Theta_{h}$ is real. Hence $-\sqrt{-1} \partial \bar{\partial} f_{*}\left(\left(c_{N} / q !\right) \omega^{q} \wedge \widehat{\sigma} \wedge h \overline{\widehat{\sigma}}\right)$ is

$$
\begin{aligned}
f_{*}\left(\left(c_{N} / q !\right) \omega^{q} \wedge \sqrt{-1} \Theta_{h} \wedge \widehat{\sigma} \wedge h \overline{\widehat{\sigma}}\right) & -f_{*}\left(\left(c_{n-q} / q !\right) \omega^{q} \wedge \mu \wedge h \bar{\mu} \wedge c_{m} d t \wedge d \bar{t}\right) \\
& -f_{*}\left(\left(c_{n-q} / q !\right) \omega^{q} \wedge \eta \wedge h \bar{\eta} \wedge c_{m} d t \wedge d \bar{t}\right) .
\end{aligned}
$$

By taking their values at $t=0$, we have our assertion.

\section{Normal and "primitive" sections, and the proof of Theorem 1.1}

Let $f:(X, \omega) \longrightarrow Y \subset \mathbb{C}^{m}$ and $(E, h)$ as in $\S 2.2$.II, and keep the notations in $\S 5$. 


\subsection{Effect of normality}

We control $\partial_{h} \sigma$ at one point for $[\sigma] \in H^{0}(Y, F)$, when it is normal at $t=0$. Recall $\partial_{h} \sigma=$ $\sum \mu^{j} \wedge d t_{j}$ with some $\mu^{j} \in A^{n-q, 0}(X, E)$. To go further, we need a genericity condition over the base $Y$. We will assume that the function $y \mapsto \operatorname{dim} H^{0}\left(X_{y}, \Omega_{X_{y}}^{n-q}\left(E_{y}\right)\right)$ is constant around $t=0$. This assumption implies that $f_{*} \Omega_{X / Y}^{n-q}(E)$ is locally free around $t=0$, and that the fiber $f_{*} \Omega_{X / Y}^{n-q}(E)_{y}$ coincides with $H^{0}\left(X_{y}, \Omega_{X_{y}}^{n-q}\left(E_{y}\right)\right)$ around $t=0([8,10.5 .5]$ [10, III §12]). In case $q=0$, i.e., the case in [2, $\S]$, this assumption holds true thanks to Ohsawa-Takegoshi type $L^{2}$-extension theorem [26][25][20]. Recall Corollary 4.4 that $f_{*} \Omega_{X / Y}^{n-q}(E)=F \oplus \mathcal{K}$, where $\mathcal{K}=\mathcal{K}^{n-q}=\operatorname{Ker} L_{f}^{q}$.

Lemma 6.1. - Assume that the function $y \mapsto \operatorname{dim} H^{0}\left(X_{y}, \Omega_{X_{y}}^{n-q}\left(E_{y}\right)\right)$ is constant around $t=0$. Let $[\sigma] \in H^{0}(Y, F)$ with $\partial_{h} \sigma=\sum \mu^{j} \wedge d t_{j}$, and suppose $\partial_{g}[\sigma]=0$ at $t=0$. Then all $\left.\mu^{j}\right|_{X_{0}}$ are perpendicular to $H^{0}\left(X_{0}, \Omega_{X_{0}}^{n-q}\left(E_{0}\right)\right)$.

Proof. - We will use notations in $\S 4.1$ for $\left(X_{0}, \omega_{0}\right)$ and $\left(E_{0}, h_{0}\right)$. Let $(,)_{h_{0}}$ be the inner product of $A^{n-q, 0}\left(X_{0}, E_{0}\right)$ in terms of the metrics $\omega_{0}$ and $h_{0}$ on $X_{0}$. We have $H^{0}\left(X_{0}, \Omega_{X_{0}}^{n-q}\left(E_{0}\right)\right)=F_{0} \oplus \mathcal{K}_{0}$, which is an orthogonal direct sum by our assumption and by Lemma 4.7 (3). We fix $j$. Let $\left.\mu^{j}\right|_{X_{0}}=\tau_{0}+a_{0} \in A^{n-q, 0}\left(X_{0}, E_{0}\right)$ be the Hodge decomposition of forms so that $\tau_{0} \in H^{0}\left(X_{0}, \Omega_{X_{0}}^{n-q}\left(E_{0}\right)\right)$ and $a_{0} \in \vartheta_{h_{0}} A^{n-q, 1}\left(X_{0}, E_{0}\right)$. We would like to show that $\tau_{0}=0$.

Since $\partial_{g}[\sigma]=0$ at $t=0$, namely all $\left.\mu^{k}\right|_{X_{0}}$ are perpendicular to $F_{0}$, it follows that $\tau_{0} \in \mathcal{K}_{0}$ by Lemma 4.7 (3). Then $\omega_{0}^{q} \wedge \tau_{0}=\bar{\partial} b_{0}$ for some $b_{0} \in A^{n, q-1}\left(X_{0}, E_{0}\right)$ by Lemma 4.7 (2). Combining with Lemma 4.5 that $\partial_{h_{0}}\left(\left.\mu^{j}\right|_{X_{0}}\right)=0$, we have $\left.\int_{X_{0}} \omega_{0}^{q} \wedge \mu^{j}\right|_{X_{0}} \wedge h_{0} \overline{\tau_{0}}=0$ by integration by parts as in Lemma 4.7 (3). Then $\left\|\tau_{0}\right\|_{h_{0}}^{2}=\left(\tau_{0}+a_{0}, \tau_{0}\right)_{h_{0}}=\int_{X_{0}}\left(c_{n-q} / q !\right) \omega_{0}^{q} \wedge$ $\left.\mu^{j}\right|_{X_{0}} \wedge h_{0} \overline{\tau_{0}}=0$, and hence $\tau_{0}=0$.

Lemma 6.2. - Let $[\sigma] \in H^{0}(Y, F)$ with $\partial_{h} \sigma=\sum \mu^{j} \wedge d t_{j}$, and suppose that $\left.\mu^{j}\right|_{X_{0}}$ is perpendicular to $H^{0}\left(X_{0}, \Omega_{X_{0}}^{n-q}\left(E_{0}\right)\right)$ for some $j$. Then there exists $\xi_{0}^{j} \in A^{n-q-1,0}\left(X_{0}, E_{0}\right)$ such that $\partial_{h_{0}} \xi_{0}^{j}=\left.\mu^{j}\right|_{X_{0}}$ and that $\bar{\partial} \xi_{0}^{j}$ is primitive.

Proof. - We will use notations in $\$ 4.1$ for $\left(X_{0}, \omega_{0}\right)$ and $\left(E_{0}, h_{0}\right)$. Recall Theorem 4.1 with $\operatorname{dim} Y=0$ that the Hodge $*$-operator yields an injective homomorphism $*_{0}: \mathcal{H}^{n, q}\left(X_{0}, E_{0}\right) \longrightarrow H^{0}\left(X_{0}, \Omega_{X_{0}}^{n-q}\left(E_{0}\right)\right)$.

We consider $u:=\left.\omega_{0}^{q} \wedge \mu^{j}\right|_{X_{0}} \in A^{n, q}\left(X_{0}, E_{0}\right)$, and recall $\left(c_{n-q} / q !\right) *_{0} u=\left.\mu^{j}\right|_{X_{0}}$. Let $u=a+\bar{\partial} b+\vartheta_{h_{0}} c$ be the Hodge decomposition of forms so that $a \in \mathcal{H}^{n, q}\left(X_{0}, E_{0}\right), b$ is $\vartheta_{h_{0}}$-exact, and that $c$ is $\bar{\partial}$-exact.

We first show that $\bar{\partial} b=0$. Using integration by parts and by Lemma 4.5 (3), we have $\left.\int_{X_{0}} \bar{\partial} b \wedge h_{0} \overline{\mu^{j}}\right|_{X_{0}}=0$. Since $\|\bar{\partial} b\|_{h_{0}}^{2}=(\bar{\partial} b, u)_{h_{0}}=\int_{X_{0}} \bar{\partial} b \wedge \bar{*}_{h_{0}} u$, and since the last term is $\left.c_{n-q} q ! \int_{X_{0}} \bar{\partial} b \wedge h_{0} \overline{\mu^{j}}\right|_{X_{0}}=0$, we have $\bar{\partial} b=0$.

We next show that $a=0$. Recall in general, $(v, w)_{h_{0}}=\left(*_{0} v, *_{0} w\right)_{h_{0}}$ holds for $v, w \in$ $A^{p, q}\left(X_{0}, E_{0}\right)([11,1.2 .20])$. Since $\vartheta_{h_{0}} c \in\left(\mathcal{H}^{n, q}\left(X_{0}, E_{0}\right)\right)^{\perp}$ the orthogonal complement in $A^{n, q}\left(X_{0}, E_{0}\right)$, we have $*_{0}\left(\vartheta_{h_{0}} c\right) \in\left(*_{0} \mathcal{H}^{n, q}\left(X_{0}, E_{0}\right)\right)^{\perp}$. We also have $*_{0} u=\left.c_{n-q}^{-1} q ! \mu^{j}\right|_{X_{0}} \in$ $H^{0}\left(X_{0}, \Omega_{X_{0}}^{n-q}\left(E_{0}\right)\right)^{\perp} \subset\left(*_{0} \mathcal{H}^{n, q}\left(X_{0}, E_{0}\right)\right)^{\perp}$. On the other hand $*_{0} a \in *_{0} \mathcal{H}^{n, q}\left(X_{0}, E_{0}\right)$, hence the both sides of $*_{0} a=*_{0} u-*_{0}\left(\vartheta_{h_{0}} c\right)$ have to be 0 . 
Now we had $u=\vartheta_{h_{0}} c$ for a $\bar{\partial}$-exact form $c \in A^{n, q+1}\left(X_{0}, E_{0}\right)$. By the Lefschetz isomorphism on forms $([11,1.2 .30])$, there exists $\xi \in A^{n-q-1,0}\left(X_{0}, E_{0}\right)$ such that $\omega_{0}^{q+1} \wedge \xi=c$. We have $\omega_{0}^{q+1} \wedge \bar{\partial} \xi=\bar{\partial} c=0$, namely $\bar{\partial} \xi$ is primitive. We also have $*_{0} c=*_{0}\left(\omega_{0}^{q+1} \wedge \xi\right)=$ $c_{n-q-1}^{-1}(q+1) ! \xi$ by Lemma 2.1. Then $\left.\mu^{j}\right|_{X_{0}}=\left(c_{n-q} / q !\right) *_{0} u=\left(c_{n-q} / q !\right)\left(-*_{0} \circ *_{0}\right.$ $\left.\partial_{h_{0}}\left(*_{0} c\right)\right)=-(-1)^{n-q}\left(c_{n-q} / q !\right) \partial_{h_{0}}\left(c_{n-q-1}^{-1}(q+1) ! \xi\right)=-\sqrt{-1}(q+1) \partial_{h_{0}} \xi$. We finally take $\xi_{0}=-\sqrt{-1}(q+1) \xi$.

\subsection{Existence of strongly normal and "primitive" sections}

Here we state a key result for the curvature estimate of our Hodge metric, as a consequence of Lemma 3.1 and Lemma 6.2. The part (I) of Proposition 6.3 below in fact holds not only for $F$, but also for any locally free subsheaf of $f_{*} \Omega_{X / Y}^{n-q}(E)$. The property (3) (respectively, (4)) below will be referred to as "primitive" (respectively, strongly normal) at $t=0$.

Proposition 6.3 (cf. [2, Proposition 4.2]). - Let $\sigma^{0} \in F_{0}$ be a vector at $t=0$.

(I) Then, there exists $\sigma \in A^{n-q, 0}(X, E)$ such that $[\sigma] \in H^{0}(Y, F)$ with the following three properties: (1) $\left.\sigma\right|_{X_{0}}=\sigma^{0}$,

(2) $\partial_{g}[\sigma]=0$ at $t=0$,

(3) $\left.\eta^{j}\right|_{X_{0}} \wedge \omega_{0}^{q+1}=0$ for any $j$, where $\bar{\partial} \sigma=\sum \eta^{j} \wedge d t_{j}$.

(II) If the function $y \mapsto \operatorname{dim} H^{0}\left(X_{y}, \Omega_{X_{y}}^{n-q}\left(E_{y}\right)\right)$ is constant around $t=0$, one can take $\sigma$ in (I) with the following additional fourth property:

(4) $\left.\mu^{j}\right|_{X_{0}}=0$ for any $j$, where $\partial_{h} \sigma=\sum \mu^{j} \wedge d t_{j}$.

Proof. - (I) A local extension as in (1) and (2) is possible for any Hermitian vector bundle. Hence we start with a local extension $[\sigma] \in H^{0}(Y, F)$ satisfying (1) and (2). We write $\bar{\partial} \sigma=$ $\sum \eta^{j} \wedge d t_{j}$. By Lemma 3.1 (4), we have $\left.\left(\eta^{j} \wedge \omega^{q+1}\right)\right|_{X_{0}}=\bar{\partial} a_{0}^{j}$ for some $a_{0}^{j} \in A^{n, q+1}\left(X_{0}, E_{0}\right)$. By the Lefschetz isomorphism on forms ([11, 1.2.30]), we can write $a_{0}^{j}=b_{0}^{j} \wedge \omega_{0}^{q+1}$ for some $b_{0}^{j} \in A^{n-q-1,0}\left(X_{0}, E_{0}\right)$. We take smooth extensions $b^{j} \in A^{n-q-1,0}(X, E)$ so that $\left.b^{j}\right|_{X_{0}}=$ $b_{0}^{j}$, and we let $\widetilde{\sigma}=\sigma-\sum b^{j} \wedge d t_{j} \in A^{n-q, 0}(X, E)$. We check $[\widetilde{\sigma}]$ is what we are looking for. Since $[\widetilde{\sigma}]=[\sigma]$ in $A^{n-q, 0}(X / Y, E)$, we see $[\widetilde{\sigma}] \in H^{0}(Y, F)$, and (1) and (2) for $\widetilde{\sigma}$. Moreover $\bar{\partial} \widetilde{\sigma}=\sum\left(\eta^{j}-\bar{\partial} b^{j}\right) \wedge d t_{j}$, and $\left.\left(\eta^{j}-\bar{\partial} b^{j}\right)\right|_{X_{0}} \wedge \omega_{0}^{q+1}=\bar{\partial} a_{0}^{j}-\bar{\partial}\left(b_{0}^{j} \wedge \omega_{0}^{q+1}\right)=0$. Hence we have $\left.\eta^{j}(\widetilde{\sigma})\right|_{X_{0}} \wedge \omega_{0}^{q+1}=0$, i.e., (3) for $\widetilde{\sigma}$.

(II) We assume that the function $y \mapsto \operatorname{dim} H^{0}\left(X_{y}, \Omega_{X_{y}}^{n-q}\left(E_{y}\right)\right)$ is constant around $t=0$. We take $\sigma \in A^{n-q, 0}(X, E)$ which satisfies all three properties in (I). We write $\partial_{h} \sigma=\sum \mu^{j} \wedge d t_{j}$ and $\bar{\partial} \sigma=\sum \eta^{j} \wedge d t_{j}$. By Lemma 6.1 and 6.2, for every $j$, there exists $\xi_{0}^{j} \in A^{n-q-1,0}\left(X_{0}, E_{0}\right)$ such that $\partial_{h_{0}} \xi_{0}^{j}=\left.\mu^{j}\right|_{X_{0}}$ and that $\bar{\partial} \xi_{0}^{j}$ is primitive. We take $\xi^{j} \in A^{n-q-1,0}(X, E)$ such that $\left.\xi^{j}\right|_{X_{0}}=\xi_{0}^{j}$ for every $j$. We consider $\widetilde{\sigma}=\sigma-\sum_{j} \xi^{j} \wedge d t_{j}$. Since $[\widetilde{\sigma}]=[\sigma]$ in $A^{n-q, 0}(X, E)$, we see $[\widetilde{\sigma}] \in H^{0}(Y, F)$, and (1) and (2) for $\widetilde{\sigma}$. We have $\partial_{h} \widetilde{\sigma}=\sum\left(\mu^{j}-\partial_{h} \xi^{j}\right) \wedge d t_{j}$ and $\bar{\partial} \widetilde{\sigma}=\sum\left(\eta^{j}-\bar{\partial} \xi^{j}\right) \wedge d t_{j}$. The property (3) for $\widetilde{\sigma}$ follows from the primitivity of $\left.\eta^{j}\right|_{X_{0}}$ for $\sigma$ and of $\left.\left(\bar{\partial} \xi^{j}\right)\right|_{X_{0}}=\bar{\partial} \xi_{0}^{j}$. The property (4) for $\tilde{\sigma}$ follows from $\left.\mu^{j}\right|_{X_{0}}-\left.\left(\partial_{h} \xi^{j}\right)\right|_{X_{0}}=0$. 


\subsection{Nakano semi-positivity}

Proposition 6.4. - Let $\sigma_{1}, \ldots, \sigma_{m} \in A^{n-q, 0}(X, E)$ with $\left[\sigma_{1}\right], \ldots,\left[\sigma_{m}\right] \in H^{0}(Y, F)$ and satisfying the properties (3) and (4) in Proposition 6.3. Then $\sqrt{-1} \partial \bar{\partial} T([\sigma])_{t=0} \leq 0$ in Notation 5.7 for these $\sigma_{1}, \ldots, \sigma_{m}$.

Proof. - We will use the notations in 5.7. By the property (4) in Proposition 6.3, we have $\left.\mu\right|_{X_{0}}=0$. The property (3) in Proposition 6.3 implies that $\left.\eta\right|_{X_{0}}$ is primitive. In particular, by using Lemma 2.1, $-\left.\int_{X_{0}}\left(c_{n-q} / q !\right)\left(\omega^{q} \wedge \eta \wedge h \bar{\eta}\right)\right|_{X_{0}}=\left\|\left.\eta\right|_{X_{0}}\right\|_{h_{0}}^{2}$ the square norm with respect to $\omega_{0}$ and $h_{0}$. Then the formula in Lemma 5.8 is

$$
-\sqrt{-1} \partial \bar{\partial} T([\sigma])_{t=0}=f_{*}\left(\left(c_{N} / q !\right) \omega^{q} \wedge \sqrt{-1} \Theta_{h} \wedge \widehat{\sigma} \wedge h \overline{\widehat{\sigma}}\right)_{t=0}+\left\|\left.\eta\right|_{X_{0}}\right\|_{h_{0}}^{2} .
$$

The right hand side is non-negative, since the curvature $\Theta_{h}$ is Nakano semi-positive.

Corollary 6.5. - $(F, g)$ is Nakano semi-positive, and hence so is $R^{q} f_{*} \Omega_{X / Y}^{n}(E)$.

Proof. - Since $g$ is a smooth Hermitian metric of $F$, to show the Nakano semipositivity, it is enough to show it on the complement of an analytic subset of $Y$. By Grauert ([8, 10.5.4][10, III.12.8, 12.9]), there exists an analytic subset $Z \subset Y$ such that the function $y \mapsto \operatorname{dim} H^{0}\left(X_{y}, \Omega_{X_{y}}^{n-q}\left(E_{y}\right)\right)$ is constant on $Y \backslash Z$. We apply the criterion in Lemma 5.6 at each point on $Y \backslash Z$. Then thanks to Proposition 6.3, Proposition 6.4 in fact shows that $g$ is Nakano semi-positive on $Y \backslash Z$.

\section{REFERENCES}

[1] C. Bănică, O. Stănăsila, Algebraic methods in the global theory of complex spaces, Editura Academiei, 1976.

[2] B. Berndtsson, Curvature of vector bundles associated to holomorphic fibrations, preprint arXiv:math.CV/0511225v2, to appear in Ann. of Math.

[3] B. Berndtsson, M. PăUn, Bergman kernels and the pseudoeffectivity of relative canonical bundles, Duke Math J. 145 (2008), 341-378.

[4] I. ENOKI, Kawamata-Viehweg vanishing theorem for compact Kähler manifolds, in Einstein metrics and Yang-Mills connections (Sanda, 1990), Lecture Notes in Pure and Appl. Math., 145, Dekker, 1993, 59-68.

[5] H. Esnault, E. Viehweg, Lectures on vanishing theorems, DMV Seminar, 20, Birkhäuser, 1992.

[6] O. Fujino, Higher direct images of log canonical divisors, J. Differential Geom. 66 (2004), 453-479.

[7] T. Fujita, On Kähler fiber spaces over curves, J. Math. Soc. Japan 30 (1978), 779-794.

[8] H. Grauert, R. Remmert, Coherent analytic sheaves, Grund. Math. Wiss., 265, Springer, 1984.

[9] P. A. Griffiths, Periods of integrals on algebraic manifolds. III. Some global differential-geometric properties of the period mapping, Publ. Math. I.H.É.S. 38 (1970), 125-180.

[10] R. Hartshorne, Algebraic geometry, Graduate Texts in Math., 52, Springer, 1977.

$4^{\mathrm{e}}$ SÉRIE - TOME $41-2008-\mathrm{N}^{\mathrm{o}} 6$ 
[11] D. Huybrechts, Complex geometry, Universitext, Springer, 2005.

[12] Y. Kawamata, Characterization of abelian varieties, Compositio Math. 43 (1981), 253276.

[13] Y. Kawamata, Subadjunction of log canonical divisors. II, Amer. J. Math. 120 (1998), 893-899.

[14] K. Kodaira, Complex manifolds and deformation of complex structures, Grund. Math. Wiss., 283, Springer, 1986.

[15] J. Kollár, Higher direct images of dualizing sheaves. I, Ann. of Math. 123 (1986), 1142.

[16] J. Kollár, Higher direct images of dualizing sheaves. II, Ann. of Math. 124 (1986), $171-202$.

[17] J. Kollár, Kodaira's canonical bundle formula and adjunction, in Flips for 3-folds and 4-folds (A. Corti, ed.), 2007.

[18] N. Levenberg, H. Yamaguchi, The metric induced by the Robin function, Mem. Amer. Math. Soc. 92 (1991), 156.

[19] F. Maitani, H. Yamaguchi, Variation of Bergman metrics on Riemann surfaces, Math. Ann. 330 (2004), 477-489.

[20] L. Manivel, Un théorème de prolongement $L^{2}$ de sections holomorphes d'un fibré hermitien, Math. Z. 212 (1993), 107-122.

[21] S. Mori, Classification of higher-dimensional varieties, in Algebraic geometry, Bowdoin, 1985 (Brunswick, Maine, 1985), Proc. Sympos. Pure Math., 46, Amer. Math. Soc., 1987, 269-331.

[22] A. Moriwaki, Torsion freeness of higher direct images of canonical bundles, Math. Ann. 276 (1987), 385-398.

[23] C. Mourougane, Images directes de fibrés en droites adjoints, Publ. Res. Inst. Math. Sci. 33 (1997), 893-916.

[24] C. Mourougane, S. Takayama, Hodge metrics and positivity of direct images, J. reine angew. Math. 606 (2007), 167-178.

[25] T. Ohsawa, On the extension of $L^{2}$ holomorphic functions. II, Publ. Res. Inst. Math. Sci. 24 (1988), 265-275.

[26] T. Ohsawa, K. Takegoshi, On the extension of $L^{2}$ holomorphic functions, Math. $Z$. 195 (1987), 197-204.

[27] K. Takegoshi, Higher direct images of canonical sheaves tensorized with semipositive vector bundles by proper Kähler morphisms, Math. Ann. 303 (1995), 389416.

[28] H. Tsusi, Variation of Bergman kernels of adjoint line bundles, preprint arXiv:math.CV/0511342.

[29] E. VieHWEG, Weak positivity and the additivity of the Kodaira dimension for certain fibre spaces, in Algebraic varieties and analytic varieties (Tokyo, 1981), Adv. Stud. Pure Math. 1 (1983), 329-353.

[30] E. Vienweg, Quasi-projective moduli for polarized manifolds, Ergeb. Math. und ihrer Grenzgebiete, 30, Springer, 1995. 
[31] C. Voisin, Théorie de Hodge et géométrie algébrique complexe, Cours Spécialisés, 10, Soc. Math. de France, 2002, version anglaise : Hodge Theory and Complex algebraic geometry I and II, Cambridge Studies in advanced Mathematics 76 et 77.

[32] H. Yamaguchi, Variations of pseudoconvex domains over $\mathbb{C}^{n}$, Michigan Math. J. 36 (1989), 415-457.

(Manuscrit reçu le 29 août 2007;

accepté, après révision, le 13 mai 2008.)

\author{
Christophe Mourougane \\ Université Rennes I \\ Institut de Recherche Mathématique de Rennes \\ Campus de Beaulieu \\ 35042 Rennes Cedex, France \\ E-mail: christophe.mourougane@univ-rennes1.fr \\ Shigeharu TAKaYAma \\ Graduate School of Mathematical Sciences \\ University of Tokyo \\ 3-8-1 Komaba \\ Tokyo 153-8914, Japan \\ E-mail: taka@ms.u-tokyo.ac.jp
}

